\title{
GEOMETRIC ASPECTS OF HIGH-ORDER EIGENVALUE PROBLEMS I. STRUCTURES ON SPACES OF BOUNDARY CONDITIONS
}

\author{
XIFANG CAO and HONGYOU WU
}

Received 11 March 2002 and in revised form 3 November 2003

\begin{abstract}
We consider some geometric aspects of regular eigenvalue problems of an arbitrary order. First, we clarify a natural geometric structure on the space of boundary conditions. This structure is the base for studying the dependence of eigenvalues on the boundary condition involved, and reveals new properties of these eigenvalues. Then, we solve the selfadjointness condition explicitly and obtain a manifold structure on the space of selfadjoint boundary conditions and several other consequences. Moreover, we give complete characterizations of several subsets of boundary conditions such as the set of all complex boundary conditions having a given complex number as an eigenvalue, and describe some of them topologically. The shapes of some of these subsets are shown to be independent of the quasidifferential equation in question.
\end{abstract}

2000 Mathematics Subject Classification: 34B05, 34L05.

1. Introduction. The study of regular spectral problems for linear ordinary differential equations (more generally, quasidifferential equations, to be abbreviated as QDEs) originated from a series of seminal papers of Sturm and Liouville in 1836/1837, while the singular case started with the celebrated work of Weyl in 1910 introducing the limit-point and limit-circle dichotomy. Another important milestone in this area is the Glazman-Krein-Naĭmark (GKN) theorem [18] of 1950, see also [4, 7, 8, 20] for generalizations (which will be included in the theorem). This theorem gives a one-to-one correspondence between the selfadjoint differential operators in a Hilbert function space representing a given QDE and the unitary isometries on an appropriate finitedimensional subspace (or equivalently, certain Lagrange subspaces of some finitedimensional quotient space, see [5, 6]). In the regular case and some subcases of the singular case, the GKN theorem also yields a characterization of the selfadjoint operators in terms of (linear) complex boundary conditions (BCs).

In this paper, we only consider regular problems. In this case, the GKN characterization of selfadjointness in terms of a complex BC can be simply expressed as the algebraic condition

$$
A E \overline{A^{t}}=B E \overline{B^{t}}
$$

where $E$ is a fixed matrix, see (2.23), while $A$ and $B$ come from the coefficient matrix $(A \mid B)$ of the BC. Note that the coefficient matrix $(A \mid B)$ is unique only up to left multiplications by nonsingular $n \times n$ complex matrices, where $n$ is the order of the 
QDE. If $n=2$, then $(A \mid B)$ can be chosen to equal

$$
\left(\begin{array}{cccc}
\cos \alpha & -\sin \alpha & 0 & 0 \\
0 & 0 & \cos \beta & -\sin \beta
\end{array}\right)
$$

with $\alpha \in[0, \pi)$ and $\beta \in(0, \pi]$, or

$$
\left(\begin{array}{cccc}
e^{i \gamma} k_{11} & e^{i \gamma} k_{12} & -1 & 0 \\
e^{i \gamma} k_{21} & e^{i \gamma} k_{22} & 0 & -1
\end{array}\right)
$$

with $\gamma \in[0, \pi), k_{11}, k_{12}, k_{21}, k_{22} \in \mathbb{R}$, and $k_{11} k_{22}-k_{12} k_{21}=1$. Since the determinant 1 condition $k_{11} k_{22}-k_{12} k_{21}=1$ can be explicitly solved, we see that the selfadjoint BCs of order 2 are explicitly given by (1.2) and (1.3). It seems to us that order 2 is the only order where the selfadjoint BCs are all explicitly known.

For some purposes (such as the situations where one wants to use all the selfadjoint $\mathrm{BC}$ close to a given selfadjoint $\mathrm{BC}$ in some sense), the forms of selfadjoint BCs given by (1.2) and (1.3) are not convenient. The reason is very simple: selfadjoint BCs of the form (1.3) can be close to a selfadjoint BC of the form (1.2). Actually, in [1, 9, 12, 14], the subset

$$
\left\{\left(\begin{array}{cccc}
1 & r & 0 & \bar{z} \\
0 & z & -1 & s
\end{array}\right) ; r, s \in \mathbb{R}, z \in \mathbb{C}\right\}
$$

of (coefficient matrices of) selfadjoint BCs of order 2 and three similar ones proved to be more suitable to such situations. Note that these subsets are open under the topology on the set $\mathscr{B}_{2}^{\mathbb{C}}$ of all selfadjoint BCs of order 2 induced from the Grassmann manifold topology on the set of all complex BCs of order 2, each of them can be identified with $\mathbb{R}^{4}$, and they together form an atlas of coordinate charts on $\mathscr{B}_{2}^{\mathbb{C}}$ (and hence $\mathscr{B}_{2}^{\mathbb{C}}$ is a real-analytic manifold).

One of the main results of this paper is a successful generalization of the subsets of selfadjoint BCs of the form in (1.4) to an arbitrary order, see Lemmas 3.10 and 3.17 together with Lemma 3.8, (3.22), and (3.45). For example, any selfadjoint BC of order 3 is in the open set

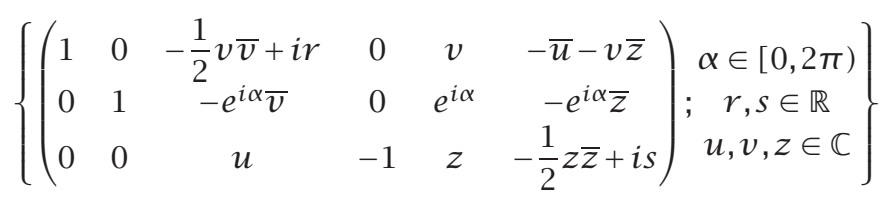

or one of three similar open sets. In this way, we not only explicitly solve the selfadjointness condition (1.1), but also obtain a manifold structure on the space of selfadjoint BCs (see Theorems 3.11, 3.15, 3.18, and 3.21). To the best of our knowledge, for the orders greater than or equal to 3 , the selfadjointness condition is explicitly solved for the first time. We believe that this explicit representation of the selfadjoint BCs is an important preparation for an in-depth study of the dependence of the eigenvalues of a selfadjoint problem on its $\mathrm{BC}$ for the orders greater than or equal to 3 (see, e.g., $[1,3,9,12,14]$ for order 2), and we plan to undertake this task in forthcoming publications. 
We would like to mention here that by a dimension count, a selfadjoint operator corresponding to the QDE is generically represented by more than one selfadjoint BC (see Remark 3.12 for details). Therefore, it is interesting to determine which selfadjoint $\mathrm{BCs}$ represent the same selfadjoint operator.

Under our explicit representation of the selfadjoint BCs, one directly sees how many algebraic equations in a selfadjoint BC can be only at the left-end point of the interval of the QDE and how many only at the right-end point. The two numbers must equal each other (see Corollaries 3.14 and 3.20). When these two numbers are fixed, one even sees the dimension of the subspace of such selfadjoint BCs. In particular, separated selfadjoint BCs only occur when the order $n$ is even. The subspace $\mathscr{R}_{n}^{\mathbb{C}, \mathrm{s}}$ of such selfadjoint BCs is a manifold of (real) dimension $n^{2} / 2$. The subspace of real separated selfadjoint BCs is a submanifold of $\mathscr{B}_{n}^{\mathbb{C}, s}$ and has a dimension of $n^{2} / 4+n / 2$. This shows that when the order is even and greater than or equal to 4 , almost all separated selfadjoint BCs are nonreal. The existence of nonreal separated selfadjoint BCs for these orders was first shown by Everitt and Markus in [5]. Using our representation of the selfadjoint $\mathrm{BCs}$, one can directly write down all these selfadjoint BCs.

Our method also applies to the singular case as long as the selfadjointness condition can be expressed as algebraic equations of a form similar to (1.1). In particular, if the two endpoints of the interval of a singular QDE are both of the limit-circle type, then the selfadjoint BCs can be explicitly written down in the same way as in the regular case. We remark that $[5,6]$ provide some new information about how many and/or what kind of algebraic equations there are in a singular selfadjoint BC.

In the last section of this paper, Section 4 , we first discuss some Lie group actions on spaces of BCs. Then, for a given QDE of order $n$ and each complex number $\lambda$, we show that there is a unique complex $\mathrm{BC}$ having $\lambda$ as an eigenvalue of geometric multiplicity $n$, and we find the BC. We also characterize the set of complex BCs having $\lambda$ as an eigenvalue and the set of real BCs having a real number $\lambda$ as an eigenvalue. The determination of these two sets uses the above Lie group actions. Moreover, we also present a topological description of each of these two sets.

2. Notation and basic results. In this section, we introduce our notation and recall some basic facts about QDEs and their boundary value problems (BVPs).

For any $n, m \in \mathbb{N}$, we use $\mathrm{M}_{n, m}(\mathbb{C})$ to denote the vector space of $n \times m$ matrices with complex entries and $\mathrm{M}_{n, m}^{*}(\mathbb{C})$ its open subset consisting of the elements with the maximum rank $\min \{n, m\}$. We define $\mathrm{M}_{n, m}(\mathbb{R})$ and $\mathrm{M}_{n, m}^{*}(\mathbb{R})$ in a similar way. When a capital Latin or Greek letter stands for a matrix, the entries of the matrix will be denoted by the corresponding lower case letter with two indices. If $A \in \mathrm{M}_{n, m}(\mathbb{C})$, then $A^{t}$ and $A^{*}$ are the transpose and the complex conjugate transpose of $A$, respectively. The general linear group $\mathrm{GL}(n, \mathbb{C}):=\mathrm{M}_{n, n}(\mathbb{C})$ is a complex Lie group under the matrix multiplication, while the special linear group $\operatorname{SL}(n, \mathbb{C})$ consists of the elements of $\operatorname{GL}(n, \mathbb{C})$ with determinant 1 and is a Lie subgroup of $\operatorname{GL}(n, \mathbb{C})$. One defines $\operatorname{GL}(n, \mathbb{R})$ and $\operatorname{SL}(n, \mathbb{R})$ similarly. Let $J$ be an open interval, bounded or unbounded. Assume that $S$ is one of the spaces $\mathbb{C}^{n}, \mathbb{R}^{n}, \mathrm{M}_{n, m}(\mathbb{C})$, and $\mathrm{M}_{n, m}(\mathbb{R})$. We denote by $\mathrm{L}(J, S)$ the space of Lebesgue integrable $S$-valued functions on $J$, and $\operatorname{AC}_{\text {loc }}(J, S)$ the space of $S$-valued functions which are absolutely continuous on all compact subintervals of $J$. 
For the rest of this paper, we fix $n \in \mathbb{N}$ satisfying $n \geq 2$ and $J=(a, b)$ with

$$
-\infty \leq a<b \leq+\infty
$$

Assume that $F \in \mathrm{L}\left(J, \mathrm{M}_{n, n}(\mathbb{C})\right)$ satisfies

$$
\begin{aligned}
f_{i, i+1} \neq 0 & \text { a.e. on } J \text { for } 1 \leq i \leq n-1, \\
f_{i, j}=0 & \text { a.e. on } J \text { for } 2 \leq i+1<j \leq n .
\end{aligned}
$$

We define $\Gamma_{0}=\mathrm{L}(J, \mathbb{C}), y^{[0]}=y$ for $y \in \Gamma_{0}$, and

$$
\begin{aligned}
\Gamma_{i} & =\left\{y \in \Gamma_{i-1} ; y^{[i-1]} \in \operatorname{AC}_{\mathrm{loc}}(J, \mathbb{C})\right\}, \\
y^{[i]} & =\frac{\left(y^{[i-1]}\right)^{\prime}-\sum_{j=1}^{i} f_{i, j} y^{[j-1]}}{f_{i, i+1}} \text { for } y \in \Gamma_{i},
\end{aligned}
$$

for $i=1,2, \ldots, n$, where $f_{n, n+1}=1$. We will set

$$
Q y=i^{n} y^{[n]} \text { for } y \in \Gamma_{n} .
$$

The expression $Q y$ is called the quasi-differential expression in $y$ associated with $F$. For $y \in \Gamma_{i}$, where $0 \leq i \leq n$, the function $y^{[i]}$ is called the $i$ th quasiderivative of $y$ associated with $F$. The subject of our study is the regular QDE

$$
Q y=\lambda w y \quad \text { on } J
$$

associated with $F$ and a weight $w \in \mathrm{L}(J, \mathbb{C})$, where $\lambda \in \mathbb{C}$ is the so-called spectral parameter. By a solution of (2.6) we mean a function $y \in \Gamma_{n}$ satisfying (2.6) a.e.

Throughout this paper, we will always assume that $F \in \mathrm{L}\left(J, \mathrm{M}_{n, n}(\mathbb{C})\right)$ satisfies (2.2); and $w \in \mathrm{L}(J, \mathbb{C})$ satisfies that

$$
w \text { is not a.e. } 0 \text { on } J \text {. }
$$

Even though (2.7) will not be needed for every result, we include it in our assumptions for the simplicity of our statements.

For any $y \in \Gamma_{n}$, we will also use the notation

$$
Y=\left(\begin{array}{c}
y^{[0]} \\
y^{[1]} \\
\vdots \\
y^{[n-1]}
\end{array}\right) .
$$

Then, (2.6) is equivalent to its matrix form

$$
Y^{\prime}=\left(F+(-i)^{n} \lambda W\right) Y \text { on } J
$$

where $W=\left(w_{i j}\right)_{n \times n}$ is the matrix such that $w_{n 1}=w$ and $w_{i j}=0$ otherwise. By the theory of first-order linear DEs, for any solution $y$ of (2.6), $Y$ has finite continuous extensions to the endpoints $a$ and $b$ (even when one or both of $a$ and $b$ are infinite). 
With the obvious topology on $[-\infty,+\infty]$, the following results are standard in the theory of first-order linear DEs.

THEOREM 2.1. (i) For any $x_{0} \in[a, b]$ and $C \in \mathbb{C}^{n}$, the differential equation (2.9) always has a unique solution $Y$ satisfying $Y\left(x_{0}\right)=C$.

(ii) As a continuous function on $[a, b]$, the above solution $Y$ depends continuously on

$$
\left(x_{0}, C\right) \in[a, b] \times \mathbb{C}^{n} .
$$

Let $\Phi(\cdot, \lambda)$ be the fundamental solution of (2.9) satisfying $\Phi(a, \cdot)=I$, where $I$ is the identity matrix. We will call $\Phi$ the principal matrix of (2.6). The following result is also standard in the theory of first-order linear DEs.

THEOREM 2.2. For any $x \in \mathbb{R}$, the entries of $\Phi(x, \lambda)$ are all entire functions of $\lambda$.

When $n$ is even, say, $n=2 k$ with $k \in \mathbb{N}$, and $J$ is finite if $k \geq 2$, there is a special case of the QDE (2.6), that is, the case studied by Naimmark [18] and by Weidmann [19], see also [2]: for any

$$
\frac{1}{f_{0}}, f_{1}, \ldots, f_{k} \in \mathrm{L}(J, \mathbb{C})
$$

if we define

$$
F=\left(\begin{array}{cccccccc}
0 & 1 & 0 & \ldots & \ldots & \ldots & \ldots & 0 \\
\vdots & \ddots & \ddots & \ddots & & & & \vdots \\
\vdots & & \ddots & 1 & \ddots & & & \vdots \\
\vdots & & & 0 & \frac{1}{f_{0}} & \ddots & & \vdots \\
\vdots & & . & f_{1} & 0 & 1 & \ddots & \vdots \\
\vdots & . & . & . & & \ddots & \ddots & 0 \\
0 & . & . & & & & \ddots & 1 \\
f_{k} & 0 & \ldots & \ldots & \ldots & \ldots & \ldots & 0
\end{array}\right),
$$

then $F$ belongs to $\mathrm{L}\left(J, \mathrm{M}_{n, n}(\mathbb{C})\right)$ and satisfies (2.2), thus, can be used as the coefficient matrix of (2.6). In this case, the quasiderivatives of $y$ associated with $F$ are given by

$$
\begin{gathered}
y^{[j]}=y^{(j)} \text { for } j=0,1, \ldots, k-1, \\
y^{[k]}=f_{0} y^{(k)}, \\
y^{[j]}=\left(y^{[j-1]}\right)^{\prime}-f_{j-k} y^{(n-j)} \text { for } j=k+1, \ldots, n .
\end{gathered}
$$


The quasi-differential expressions in $y$ associated with $F$ for $n=2, n=4$, or $n=6$ are of special interest:

$$
\begin{aligned}
& Q y=-\left(f_{0} y^{\prime}\right)^{\prime}+f_{1} y \quad \text { if } n=2, \\
& Q y=\left[\left(f_{0} y^{\prime \prime}\right)^{\prime}-f_{1} y^{\prime}\right]^{\prime}-f_{2} y \quad \text { if } n=4, \\
& Q y=\left\{\left[-\left(f_{0} y^{\prime \prime \prime}\right)^{\prime}+f_{1} y^{\prime \prime}\right]^{\prime}+f_{2} y^{\prime}\right\}^{\prime}+f_{3} y \quad \text { if } n=6,
\end{aligned}
$$

while, in general, $Q y$ is equal to

$$
(-1)^{k}\left\{\left\{\cdots\left\{\left[\left(f_{0} y^{(k)}\right)^{\prime}-f_{1} y^{(k-1)}\right]^{\prime}-f_{2} y^{(k-2)}\right\}^{\prime}-\cdots-f_{k-1} y^{\prime}\right\}^{\prime}-f_{k} y\right\} .
$$

Note that (2.11) implies that

$$
f_{0} \neq 0 \text { a.e. on } J \text {. }
$$

We now turn to the BVP consisting of the general QDE (2.6) and a (linear two-point) $\mathrm{BC}$ defined by

$$
A Y(a)+B Y(b)=0
$$

where $A, B \in \mathrm{M}_{n, n}(\mathbb{C})$ such that $(A \mid B) \in \mathrm{M}_{n, 2 n}^{*}(\mathbb{C})$. Note that equivalent linear algebraic equations of the form (2.17) define the same BC. Each value of $\lambda$ for which the QDE (2.6) has a nontrivial solution satisfying the $\mathrm{BC}$ (2.17) is called an eigenvalue of the BVP consisting of (2.6) and (2.17) and such a solution is called an eigenfunction for this eigenvalue. The vector space spanned by the eigenfunctions for an eigenvalue is the eigenspace for the eigenvalue, while the dimension of the eigenspace is called the geometric multiplicity of the eigenvalue. Since (2.6) has exactly $n$ linearly independent solutions, the geometric multiplicity of any eigenvalue is an integer not smaller than 1 and not larger than $n$.

THEOREM 2.3. A number $\lambda \in \mathbb{C}$ is an eigenvalue of the boundary value problem consisting of (2.6) and (2.17) if and only if

$$
\Delta(\lambda):=\operatorname{det}(A+B \Phi(b, \lambda))=0 .
$$

REMARK 2.4. Actually, a number $\lambda_{*} \in \mathbb{C}$ is an eigenvalue for (2.17) of geometric multiplicity $m$ if and only if $A+B \Phi\left(b, \lambda_{*}\right)$ has rank $n-m$; in this case, the eigenfunctions are $\sum_{i=1}^{n} c_{i} \phi_{1 i}\left(\cdot, \lambda_{*}\right)$, where the vector $\mathbf{c}=\left(c_{1} c_{2} \cdots c_{n}\right)^{t} \in \mathbb{C}^{n}$ varies over the nonzero solutions of

$$
(A+B \Phi(b, \lambda)) \mathbf{c}=0 .
$$

We will call the important function $\Delta$, unique up to a nonzero constant multiple, the characteristic function of the BVP consisting of (2.6) and (2.17). By Theorem 2.2, $\Delta$ is an entire function. This yields the following corollary. 
COROLLARY 2.5. There are only the following four possibilities:

(i) the problem has no eigenvalue;

(ii) the problem has a finite number of eigenvalues;

(iii) the problem has an infinite but countable number of eigenvalues, and the eigenvalues do not have any accumulation point;

(iv) every complex number is an eigenvalue.

The analytic multiplicity (or just multiplicity) of an isolated eigenvalue is the order of the eigenvalue as a zero of $\Delta$. An eigenvalue is said to be simple if it has multiplicity 1 , while the eigenvalues of multiplicity 2 are called double eigenvalues. When we count the (isolated) eigenvalues of a BVP in a domain in $\mathbb{C}$, their multiplicities will be taken into account.

Let $\|\cdot\|$ be a norm on $\mathrm{M}_{n, n}(\mathbb{C})$. A slightly different form of the following result has appeared in [13]. It is a consequence of Theorem 2.1, the entireness of $\Delta$, and Rouché's theorem in complex analysis.

THEOREM 2.6. Let $\mathscr{R} \subset \mathbb{C}$ be a bounded open set such that its boundary does not contain any eigenvalue of the boundary value problem consisting of (2.6) and (2.17), and $m \geq 0$ the number of eigenvalues of the problem in $\mathscr{R}$. Then, there exists a $\delta>0$ such that the boundary value problem consisting of (2.6) and any boundary condition

$$
C Y(a)+D Y(b)=0
$$

satisfying

$$
\|A-C\|+\|B-D\|<\delta
$$

also has exactly $m$ eigenvalues in $\mathscr{R}$.

A coefficient matrix $F$ is said to be E-symmetric if

$$
F E+E F^{*}=0,
$$

where

$$
E=\left(\begin{array}{cccc}
0 & \cdots & 0 & -1 \\
\vdots & . & (-1)^{2} & 0 \\
0 & . & . & \vdots \\
(-1)^{n} & 0 & \cdots & 0
\end{array}\right) .
$$

The BC (2.17) is said to be selfadjoint if

$$
A E A^{*}=B E B^{*} .
$$

Note that selfadjointness is well defined: the matrix $(A \mid B)$ in (2.17) satisfies (2.24) if and only if the coefficient matrix of any equivalent algebraic equation does. When $w>0$ a.e. on $J$, the symmetry condition and the selfadjointness condition guarantee that the BVP consisting of (2.6) and (2.17) can be identified with a selfadjoint operator in the 
weighted Hilbert space $\mathrm{L}^{2}(J, \mathbb{C} ; w)$, see $[17,18,19,20]$. From this we have the following theorem.

THEOREM 2.7. Assume that $F$ is E-symmetric, $w$ is positive a.e. on J, and the boundary condition (2.17) is selfadjoint. Then, the following conclusions hold.

(i) The boundary value problem consisting of (2.6) and (2.17) has a discrete spectrum consisting of an infinite but countable number of all real eigenvalues.

(ii) When the order $n \geq 2$ is odd, the spectrum is unbounded both from above and from below.

(iii) When the order $n=2 k$ with $k \in \mathbb{N}$ and the leading coefficient $f_{k, k+1}$ is positive a.e. on $J$, then the spectrum is bounded from below but not from above. Thus, the eigenvalues can be ordered into a sequence

$$
\lambda_{1} \leq \lambda_{2} \leq \lambda_{3} \leq \cdots,
$$

approaching $+\infty$ so that the number of times an eigenvalue appears in the sequence is equal to its multiplicity.

(iv) When the order $n=2 k$ with $k \in \mathbb{N}$ and the leading coefficient $f_{k, k+1}$ changes its sign on $J$, that is, both the set $\left\{x \in J, f_{k, k+1}(x)>0\right\}$ and the set $\left\{x \in J, f_{k, k+1}(x)<0\right\}$ have positive Lebesgue measures, then the spectrum is unbounded from above and from below.

3. Spaces of boundary conditions. Recall that the ( $n$th order) complex BCs are represented by systems of $n$ linearly independent homogeneous equations on $Y(a)$ and $Y(b)$ with complex coefficients. Two such systems

$$
A Y(a)+B Y(b)=0, \quad C Y(a)+D Y(b)=0
$$

represent the same complex $\mathrm{BC}$ if and only if there exists a matrix $T \in \operatorname{GL}(n, \mathbb{C})$ such that

$$
(C \mid D)=(T A \mid T B)
$$

Thus, the space $\mathscr{A}^{\mathbb{C}}$ of complex BCs is just the quotient space

$$
\operatorname{GL}(n, \mathbb{C}) \backslash \mathrm{M}_{n, 2 n}^{*}(\mathbb{C}) .
$$

Here we put $\mathrm{GL}(n, \mathbb{C})$ on the left in the quotient to indicate that the corresponding factors are on the left. The complex BC represented by the first system in (3.1) will be denoted by $[A \mid B]$. Note here that square brackets, not parentheses, are used. Usual bold-faced capital Latin letters, such as A, will also be used to denote BCs. We give the space $\mathrm{M}_{n, 2 n}(\mathbb{C})$ of $n \times 2 n$ complex matrices the usual topology on $\mathbb{C}^{n \times 2 n}$, then $\mathrm{M}_{n, 2 n}^{*}(\mathbb{C})$ is an open subset of $\mathrm{M}_{n, 2 n}(\mathbb{C})$. In this way, $\mathbb{A}^{\mathbb{C}}$ inherits a topology, the quotient topology.

THEOREM 3.1. The space $\mathbb{A}^{\mathbb{C}}$ of nth-order complex boundary conditions is a connected and compact complex manifold of complex dimension $n^{2}$.

Proof. The space $\mathscr{A}^{\mathbb{C}}$ is also the space of complex $n$-planes in $\mathbb{C}^{2 n}$ through the origin, so it is the well-known Grassmann manifold $G_{n}\left(\mathbb{C}^{2 n}\right)$, see $[10,11]$. 
REMARK 3.2. Note that $\mathscr{A}^{\mathbb{C}} \backslash\{[I \mid 0],[0 \mid-I]\}$ is not compact. This is the reason for including in $\mathscr{A}^{\mathbb{C}}$ the degenerated $B C S[I \mid 0]$ and $[0 \mid-I]$.

When $n=2, \mathscr{B}^{\mathbb{C}}$ has the following canonical atlas of local coordinate systems:

$$
\begin{aligned}
& \mathcal{N}_{1,2}^{\mathbb{C}}=\left\{[I \mid B] ; B \in \mathrm{M}_{2,2}(\mathbb{C})\right\}, \\
& \mathcal{N}_{1,3}^{\mathbb{C}}=\left\{\left[\begin{array}{cccc}
1 & a_{12} & 0 & b_{12} \\
0 & a_{22} & -1 & b_{22}
\end{array}\right] ; a_{12}, a_{22}, b_{12}, b_{22} \in \mathbb{C}\right\}, \\
& \mathcal{N}_{1,4}^{\mathbb{C}}=\left\{\left[\begin{array}{cccc}
1 & a_{12} & b_{11} & 0 \\
0 & a_{22} & b_{21} & -1
\end{array}\right] ; a_{12}, a_{22}, b_{11}, b_{21} \in \mathbb{C}\right\}, \\
& \mathcal{N}_{2,3}^{\mathbb{C}}=\left\{\left[\begin{array}{llll}
a_{11} & 1 & 0 & b_{12} \\
a_{21} & 0 & -1 & b_{22}
\end{array}\right] ; a_{11}, a_{21}, b_{12}, b_{22} \in \mathbb{C}\right\}, \\
& \mathcal{N}_{2,4}^{\mathbb{C}}=\left\{\left[\begin{array}{llll}
a_{11} & 1 & b_{11} & 0 \\
a_{21} & 0 & b_{21} & -1
\end{array}\right] ; a_{11}, a_{21}, b_{11}, b_{21} \in \mathbb{C}\right\}, \\
& \mathcal{N}_{3,4}^{\mathbb{C}}=\left\{[A \mid-I] ; A \in \mathrm{M}_{2,2}(\mathbb{C})\right\} ;
\end{aligned}
$$

when $n=3, \mathscr{B}^{\mathbb{C}}$ has the following canonical atlas of local coordinate systems:

$$
\begin{aligned}
\mathcal{N}_{1,2,3}^{\mathbb{C}} & =\left\{[I \mid B] ; B \in \mathrm{M}_{3,3}(\mathbb{C})\right\}, \\
\mathcal{N}_{1,2,4}^{\mathbb{C}} & =\left\{\left[\begin{array}{cccccc}
1 & 0 & a_{13} & 0 & b_{12} & b_{13} \\
0 & 1 & a_{23} & 0 & b_{22} & b_{23} \\
0 & 0 & a_{33} & -1 & b_{32} & b_{33}
\end{array}\right] ; \begin{array}{r}
a_{13}, a_{23}, a_{33}, \\
b_{12}, b_{13}, b_{22}, \\
b_{23}, b_{32}, b_{33} \in \mathbb{C}
\end{array}\right\}, \ldots, \\
\mathcal{N}_{4,5,6}^{\mathbb{C}} & =\left\{[A \mid-I] ; A \in \mathrm{M}_{3,3}(\mathbb{C})\right\},
\end{aligned}
$$

and so forth. Coordinate systems of the form indicated by (3.4) and (3.5) are the socalled canonical coordinate systems on $\mathbb{A}^{\mathbb{C}}$. In general, for each subset $N \subset \mathbb{N}_{2 n}$ with exactly $n$ elements, we will use $\mathcal{N}_{N}^{\mathbb{C}}$ to denote the corresponding canonical coordinate system on $\mathscr{A}^{\mathbb{C}}$.

Given a $\mathrm{BC}[A \mid B]$, one can bring it into the standard form of the elements in a canonical coordinate system using the Gauss elimination. More precisely, one applies only row operations to the matrix $(A \mid B)$ such that each of some $n$ columns has exactly one nonzero entry; all these nonzero entries in the left-half block equal 1 and are in the top rows, while the ones in the right-half block equal -1 and are in the remaining rows; and any two of these nonzero entries are in different rows. These columns of the new matrix will be called the normalized columns, while the other columns will be called the unnormalized columns. Such a procedure will be called a normalization of $[A \mid B]$.

Similarly, the space $\mathscr{A}^{\mathbb{R}}$ of ( $n$ th-order) real BCs is just

$$
\mathrm{GL}(n, \mathbb{R}) \backslash \mathrm{M}_{n, 2 n}^{*}(\mathbb{R}),
$$

and we have the following result. 
THEOREM 3.3. The space $\mathscr{A}^{\mathbb{R}}$ of nth-order real boundary conditions is a connected and compact real-analytic manifold of dimension $n^{2}$.

The space $\mathscr{A}^{\mathbb{R}}$ has an atlas of canonical coordinate systems similar to the canonical coordinate systems on $\mathscr{A}^{\mathbb{C}}$, and their notation is clear.

Under the Grassmann manifold structures on $\mathscr{A}^{\mathbb{C}}$ and $\mathscr{A}^{\mathbb{R}}$, different types of BCs (e.g., the coupled BCs, the degenerated BCs, and the separated BCs) are naturally related to each other. Moreover, by applying Theorem 2.6 to each of the canonical coordinate systems on $\mathscr{A}^{\mathbb{C}}$, one deduces the following general version of the continuous dependence of eigenvalues on $\mathrm{BC}$.

THEOREM 3.4. Let $\mathscr{R} \subset \mathbb{C}$ be a bounded open set whose boundary does not contain any eigenvalue for a boundary condition $\mathbf{A} \in \mathscr{A}^{\mathbb{C}}$, and $m \geq 0$ the number of the eigenvalues for $\mathbf{A}$ in $\mathscr{R}$. Then, there exists a neighborhood 0 of $\mathbf{A}$ in $\mathscr{A}^{\mathbb{C}}$ such that there are exactly $m$ eigenvalues in $\mathscr{R}$ for any boundary condition in $\mathbb{O}$.

REMARK 3.5. Theorem 3.4 implies that if $\lambda_{*}$ is a simple eigenvalue for a BC $\mathrm{A} \in \mathscr{A}^{\mathbb{C}}$, then there is a continuous function $\Lambda: O \rightarrow \mathbb{C}$ defined on a connected neighborhood 0 of $\mathrm{A}$ in $\mathscr{A}^{\mathbb{C}}$ such that

(i) $\Lambda(\mathrm{A})=\lambda_{*}$;

(ii) for any $\mathbf{X} \in \mathbb{O}, \Lambda(\mathbf{X})$ is a simple eigenvalue for $\mathbf{X}$.

Any two such functions agree on the common part (still a neighborhood of $\mathbf{A}$ in $\mathscr{A}^{\mathbb{C}}$ ) of their domains. So, by the continuous simple eigenvalue branch through $\lambda_{*}$ we will mean any such function.

In general, by a continuous eigenvalue branch we mean a continuous function $\Lambda$ : $\mathbb{O} \rightarrow \mathbb{C}$ defined on a connected open set $\mathbb{O} \subset \mathbb{A}^{\mathbb{C}}$ such that for each $\mathbf{A} \in \mathbb{O}, \Lambda(\mathbf{A})$ is an eigenvalue for $\mathbf{A}$.

We may restrict our attention to the space $\mathbb{A}^{\mathbb{R}}$ of real BCs. There is a result for $\mathscr{A}^{\mathbb{R}}$ similar to Theorem 3.4. Moreover, the concepts of continuous eigenvalue branch over $\mathscr{A}^{\mathbb{R}}$ and continuous simple eigenvalue branch over $\mathscr{A}^{\mathbb{R}}$ have their clear meanings.

The concept of continuous eigenvalue branch has appeared in [13, 15, 16]. The following result illustrates the importance of the concept of continuous simple eigenvalue branch in addition to implying existence of eigenvalues.

THEOREM 3.6. If the quasi-differential equation (2.6) has real coefficients, then the values of a continuous simple eigenvalue branch over $A^{\mathbb{R}}$ are either all real or all nonreal.

Proof. See [15, Theorem 3.8].

Next, we consider the selfadjoint BCs. First, we want to show that they form a closed subset of $\mathscr{A}^{\mathbb{C}}$.

LEMMA 3.7. The space $\mathscr{B}^{\mathbb{C}}$ of complex selfadjoint boundary conditions is a closed subset of $\mathscr{A}^{\mathbb{C}}$, and the space $\mathscr{B}^{\mathbb{R}}$ of real selfadjoint boundary conditions is a closed subset of $\mathscr{A}^{\mathbb{R}}$. Hence, $\mathscr{B}^{\mathbb{C}}$ and $\mathscr{B}^{\mathbb{R}}$ are compact spaces.

Proof. Let $\left\{\left[A_{i} \mid B_{i}\right] ; i \in \mathbb{N}\right\} \subset \mathscr{P}^{\mathbb{C}}$ such that $\left[A_{i} \mid B_{i}\right] \rightarrow\left[A_{\infty} \mid B_{\infty}\right] \in \mathscr{A}^{\mathbb{C}}$ as $i \rightarrow+\infty$. We can assume that $\left[A_{\infty} \mid B_{\infty}\right],\left[A_{1} \mid B_{1}\right],\left[A_{2} \mid B_{2}\right], \ldots$ all belong to the same canonical 
coordinate system on $\mathscr{A}^{\mathbb{C}}$. So, we can actually assume that $\left(A_{\infty} \mid B_{\infty}\right),\left(A_{1} \mid B_{1}\right),\left(A_{2} \mid B_{2}\right), \ldots$ are all in the standard form of that coordinate system. Thus, as $i \rightarrow+\infty, A_{i} \rightarrow A_{\infty}$ and $B_{i} \rightarrow B_{\infty}$ in $\mathrm{M}_{n, n}(\mathbb{C})$. From $A_{i} E A_{i}^{*}=B_{i} E B_{i}^{*}$ for each $i \in \mathbb{N}$, we then obtain $A_{\infty} E A_{\infty}^{*}=$ $B_{\infty} E B_{\infty}^{*}$, that is, $\left[A_{\infty} \mid B_{\infty}\right] \in \mathscr{B}^{\mathbb{C}}$. Therefore, $\mathscr{B}^{\mathbb{C}}$ is closed. Similarly, $\mathscr{B}^{\mathbb{R}}$ is also closed.

For each $m \in \mathbb{N}$, set $\mathbb{N}_{m}=\{1, \ldots, m\}$. Let $A \in \mathrm{M}_{n, n}(\mathbb{C})$ and $i \in \mathbb{N}_{n} \backslash\{(n+1) / 2\}$. We will say that $B$ 's $i$ th column and its $(n+1-i)$ th column form a symmetric pair. Denote by $A^{(i)}$ the matrix whose $i$ th column is the $(n+1-i)$ th column of $A,(n+1-i)$ th column is the $i$ th column of $A$ multiplied by $(-1)^{n+1}$, and the other columns are the same as those of $A$. The next result shows that in many canonical coordinate systems on $\mathscr{A}^{\mathbb{C}}$ checking the selfadjointness amounts to the same work.

LEMMA 3.8. Let $i \in \mathbb{N}_{n}$ such that $i \neq(n+1) / 2$. Then, a boundary condition $[A \mid B]$ is selfadjoint if and only if $\left[A^{(i)} \mid B\right]$ is, and if and only if $\left[A \mid B^{(i)}\right]$ is.

Proof. For any $m \in \mathbb{N}_{n}$, we set $m^{\prime}=n+1-m$. Now, let $j, l \in \mathbb{N}_{n}$. Then, the $(j, l)$ entry of $A E A^{*}$ is given by

$$
\begin{aligned}
& \sum_{1 \leq m \leq n}^{m \neq i, m \neq i^{\prime}}(-1)^{m^{\prime}} a_{j, m^{\prime}} \overline{a_{l, m}}+(-1)^{i^{\prime}} a_{j, i^{\prime}} \overline{a_{l, i}}+(-1)^{i} a_{j, i} \overline{a_{l, i^{\prime}}} \\
& \quad=\sum_{1 \leq m \leq n}^{m \neq i, m \neq i^{\prime}}(-1)^{m^{\prime}} a_{j, m^{\prime}}^{(i)} \overline{a_{l, m}^{(i)}}+(-1)^{i} a_{j, i^{\prime}}\left[\overline{(-1)^{n+1} a_{l, i}}\right]+(-1)^{i^{\prime}}\left[(-1)^{n+1} a_{j, i}\right] \overline{a_{l, i^{\prime}}} \\
& \quad=\sum_{m=1}^{n} a_{j, m^{\prime}}^{(i)} \overline{a_{l, m}^{(i)}},
\end{aligned}
$$

which equals the $(j, l)$-entry of $A^{(i)} E\left(A^{(i)}\right)^{*}$. Therefore, $A E A^{*}=A^{(i)} E\left(A^{(i)}\right)^{*}$, and hence $[A \mid B]$ is selfadjoint if and only if $\left[A^{(i)} \mid B\right]$ is. Similarly, $[A \mid B]$ is selfadjoint if and only if $\left[A \mid B^{(i)}\right]$ is.

For illustration, we list the following applications of Lemma 3.8: when $n=3$,

$$
\begin{aligned}
& {\left[\begin{array}{llllll}
1 & 0 & a_{13} & b_{11} & b_{12} & 0 \\
0 & 1 & a_{23} & b_{21} & b_{22} & 0 \\
0 & 0 & a_{33} & b_{31} & b_{32} & -1
\end{array}\right] \in \mathscr{B}^{\mathbb{C}} \Longleftrightarrow\left[\begin{array}{cccccc}
1 & 0 & a_{13} & 0 & b_{12} & b_{11} \\
0 & 1 & a_{23} & 0 & b_{22} & b_{21} \\
0 & 0 & a_{33} & -1 & b_{32} & b_{31}
\end{array}\right] \in \mathscr{B}^{\mathbb{C}},} \\
& {\left[\begin{array}{ccccccc}
a_{11} & 0 & 1 & 0 & b_{12} & b_{13} \\
a_{21} & 1 & 0 & 0 & b_{22} & b_{23} \\
a_{31} & 0 & 0 & -1 & b_{32} & b_{33}
\end{array}\right] \in \mathscr{B}^{\mathbb{C}} \Longleftrightarrow\left[\begin{array}{cccccc}
1 & 0 & a_{11} & 0 & b_{12} & b_{13} \\
0 & 1 & a_{21} & 0 & b_{22} & b_{23} \\
0 & 0 & a_{31} & -1 & b_{32} & b_{33}
\end{array}\right] \in \mathscr{B}^{\mathbb{C}},} \\
& {\left[\begin{array}{ccccccc}
a_{11} & 0 & 1 & b_{11} & b_{12} & 0 \\
a_{21} & 1 & 0 & b_{21} & b_{22} & 0 \\
a_{31} & 0 & 0 & b_{31} & b_{32} & -1
\end{array}\right] \in \mathscr{B}^{\mathbb{C}} \Longleftrightarrow\left[\begin{array}{cccccc}
1 & 0 & a_{11} & 0 & b_{12} & b_{11} \\
0 & 1 & a_{21} & 0 & b_{22} & b_{21} \\
0 & 0 & a_{31} & -1 & b_{32} & b_{31}
\end{array}\right] \in \mathscr{B}^{\mathbb{C}},}
\end{aligned}
$$


and when $n=4$,

$$
\begin{aligned}
& {\left[\begin{array}{cccccccc}
1 & 0 & a_{13} & a_{14} & 0 & b_{12} & 0 & b_{14} \\
0 & 1 & a_{23} & a_{24} & 0 & b_{22} & 0 & b_{24} \\
0 & 0 & a_{33} & a_{34} & -1 & b_{32} & 0 & b_{34} \\
0 & 0 & a_{43} & a_{44} & 0 & b_{42} & -1 & b_{44}
\end{array}\right] \in \mathscr{B}^{\mathbb{C}}} \\
& \Longleftrightarrow\left[\begin{array}{cccccccc}
1 & 0 & a_{13} & a_{14} & 0 & 0 & -b_{12} & b_{14} \\
0 & 1 & a_{23} & a_{24} & 0 & 0 & -b_{22} & b_{24} \\
0 & 0 & a_{33} & a_{34} & -1 & 0 & -b_{32} & b_{34} \\
0 & 0 & a_{43} & a_{44} & 0 & -1 & -b_{42} & b_{44}
\end{array}\right] \in \mathscr{B}^{\mathbb{C}}, \\
& {\left[\begin{array}{cccccccc}
1 & a_{12} & 0 & a_{14} & b_{11} & b_{12} & 0 & 0 \\
0 & a_{22} & 1 & a_{24} & b_{21} & b_{22} & 0 & 0 \\
0 & a_{32} & 0 & a_{34} & b_{31} & b_{32} & 0 & -1 \\
0 & a_{42} & 0 & a_{44} & b_{41} & b_{42} & -1 & 0
\end{array}\right] \in \mathscr{B}^{\mathbb{C}}} \\
& \Longleftrightarrow\left[\begin{array}{cccccccc}
1 & 0 & -a_{12} & a_{14} & 0 & 0 & -b_{12} & -b_{11} \\
0 & 1 & -a_{22} & a_{24} & 0 & 0 & -b_{22} & -b_{21} \\
0 & 0 & -a_{32} & a_{34} & -1 & 0 & -b_{32} & -b_{31} \\
0 & 0 & -a_{42} & a_{44} & 0 & -1 & -b_{42} & -b_{41}
\end{array}\right] \in \mathscr{B}^{\mathbb{C}},
\end{aligned}
$$

and so forth.

We will see that the space of selfadjoint BCs in the even-order case is different from that in the odd-order case. For example, the space of real selfadjoint BCs is connected in the even-order case, while it has two connected components in the odd-order case. So, now, we discuss these two cases separately.

SELFADJOINT BOUNDARY CONDITIONS OF AN EVEN ORDER. Let $n=2 k$ with $k \in \mathbb{N}$. First, we have a fact on the ranks of the left- and right-half blocks in a selfadjoint BC.

LEMMA 3.9. For any $[A \mid B] \in \mathscr{S}^{\mathbb{C}}, \operatorname{rank} A \geq k$ and $\operatorname{rank} B \geq k$.

Proof. Let $l=\operatorname{rank} A$, then $l \geq 1$ since $\operatorname{rank}(A \mid B)=n$ and $|\operatorname{det} A|=|\operatorname{det} B|$. Assume that $l<k$, then we can assume further that the last $n-l$ rows of $A$ are 0 . Thus,

$$
A E A^{*}=\left(\begin{array}{ll}
C & 0 \\
0 & 0
\end{array}\right)=B E B^{*}
$$

for some $l \times l$ matrix $C$, and the last $n-l$ rows of $B$ are linearly independent (so, $\operatorname{rank} B \geq$ $n-l$ ). Normalize $[A \mid B]$ so that $n-l$ columns of $B$ are normalized. Note that after this normalization, the last $n-l$ rows of $A$ can remain 0 , which will be assumed. We normalize $[A \mid B]$ such that the number of symmetric pairs of unnormalized columns in $B$ is minimum. Since $n-l>k$, there is an integer $j \in \mathbb{N}_{k}$ such that both $B$ 's $j$ th column and its $(n+1-j)$ th column are normalized. We can assume that

$$
b_{k, j}=b_{n, n+1-j}=-1 .
$$

For each integer $m \in \mathbb{N}_{n} \backslash\{j, n+1-j\}$, we have the following: if one of $B$ 's $m$ th column and its $(n+1-m)$ th column is normalized, then either $b_{k, n+1-m}=0$ or $b_{n, m}=0$; if both 
of these two columns are unnormalized, then we must have $b_{n, m}=0$ by our choice of the normalization. Thus, the $(k, n)$-entry of $B E B^{*}$ is given by

$$
\sum_{m=1}^{n}(-1)^{n+1-m} b_{k, n+1-m} \overline{b_{n, m}}=(-1)^{j} b_{k, j} \overline{b_{n, n+1-j}}=(-1)^{j},
$$

contradicting (3.10). Therefore, $\operatorname{rank} A \geq k$. Similarly, $\operatorname{rank} B \geq k$.

Direct calculations show that the selfadjointness equation $A E A^{*}=B E B^{*}$ can be easily solved (which we will do in general soon) if every symmetric pair of columns in $A$ or in $B$ has one (and hence only one) normalized column. Naturally, one wonders if every selfadjoint $\mathrm{BC}$ can be normalized into such a form. The next result gives an affirmative answer.

LEMMA 3.10. Every $[A \mid B] \in \mathscr{B}^{\mathbb{C}}$ has a normalization such that each symmetric pair of columns in A or in B has one (and hence only one) normalized column.

Proof. Since rank $A \geq k$, we can begin the normalization of $[A \mid B]$ by normalizing $k$ columns of $A$. Note that the nonzero entries of these columns are in the first $k$ rows. We can assume that the number of symmetric pairs of unnormalized columns in $A$ is minimum. If that minimum number is 0 , then for each integer $j \in \mathbb{N}_{k}$, one of $A$ 's $j$ th column and its $(n+1-j)$ th column is normalized, and hence

$$
A E A^{*}=\left(\begin{array}{cc}
C & D \\
H & 0
\end{array}\right)
$$

for some $k \times k$ matrices $C, D$, and $H$. Otherwise, for each integer $j \in \mathbb{N}_{k}$ such that both $A$ 's $j$ th column and its $(n+1-j)$ th column are unnormalized, we must have

$$
a_{i, j}=a_{i, n+1-j}=0
$$

for $i=k+1, k+2, \ldots, n$, and hence (3.13) also holds. Thus, we can always assume that (3.13) holds.

Note that the last row of $A E A^{*}$ is just a rearrangement of the last row of $A$, with some entries signs changed. If the lower half of $B$ now has a rank less than $k$, then we can assume that the last row of $B$ is 0 , and hence so is $B E B^{*}$. Then, $A E A^{*}=B E B^{*}$ implies that the last row of $A$ is also 0 , which is impossible. So, the rows in the lower half of $B$ are linearly independent.

We now continue the normalization of $[A \mid B]$ by normalizing exactly $k$ columns in $B$ without destroying the $k$ normalized columns in $A$ used above and such that the number of symmetric pairs of unnormalized columns in $B$ is minimum. If the minimum number is not 0 , then $k \geq 2$ and, for some integer $m \in \mathbb{N}_{k}$, both $B$ 's $m$ th column and its $(n+1-m)$ th column are normalized. Hence, we can assume that

$$
b_{k+1, m}=b_{n, n+1-m}=-1 \text {. }
$$

Thus, we must have $b_{k+1, j}=0$ for each $j \neq m$ such that $B$ 's $j$ th column is normalized, 
$b_{n, j}=0$ for each $j \neq n+1-m$ such that $B$ 's $j$ th column is normalized, and $b_{k+1, j}=$ $b_{k+1, n+1-j}=0$ for each integer $j \in \mathbb{N}_{k}$ such that both $B$ 's $j$ th column and its $(n+1-j)$ th column are unnormalized. So the $(k+1, n)$-entry of $B E B^{*}$ is $(-1)^{m}$. By (3.13), $A E A^{*} \neq$ $B E B^{*}$, contradicting the selfadjointness. Therefore, after our normalization, $[A \mid B]$ does not have any symmetric pair of unnormalized columns in $B$.

Similarly, $A$ does not have any symmetric pair of unnormalized columns after the above normalization of $[A \mid B]$.

A subset $N \subset \mathbb{N}_{2 n}$ with exactly $n$ elements is called a preferred subset if for any $i \in \mathbb{N}_{k}$, exactly one of $i$ and $n+1-i$ is in $N$ and exactly one of $n+i$ and $2 n+1-i$ is in $N$. If $N$ is a preferred subset of $\mathbb{N}_{2 n}$, then the canonical coordinate system $\mathcal{N}_{N}^{\mathbb{C}}$ on $\mathscr{A}^{\mathbb{C}}$ is called a preferred coordinate system. For example, when $n=2, \mathcal{N}_{1,3}^{\mathbb{C}}, \mathcal{N}_{1,4}^{\mathbb{C}}, \mathcal{N}_{2,3}^{\mathbb{C}}$, and $\mathcal{N}_{2,4}^{\mathbb{C}}$ are preferred, while $\mathcal{N}_{1,2}^{\mathbb{C}}$ and $\mathcal{N}_{3,4}^{\mathbb{C}}$ are not. With this concept in mind, Lemma 3.10 just says that every selfadjoint BC is in a preferred coordinate system on $\mathscr{A}^{\mathbb{C}}$.

By Lemma 3.8, solving the selfadjointness equation $A E A^{*}=B E B^{*}$ in any preferred coordinate system on $\mathscr{A}^{\mathbb{C}}$ is equivalent to solving it in $\mathcal{N}_{\mathbb{C}}^{\mathbb{C}}$, where

$$
\llbracket=\{1,2, \ldots, k, n+1, n+2, \ldots, 3 k\} .
$$

If we set

$$
A=\left(\begin{array}{ll}
I & A_{\mathrm{U}} \\
0 & A_{\mathrm{L}}
\end{array}\right), \quad B=\left(\begin{array}{cc}
0 & B_{\mathrm{U}} \\
-I & B_{\mathrm{L}}
\end{array}\right),
$$

where $A_{\mathrm{U}}, A_{\mathrm{L}}, B_{\mathrm{U}}, B_{\mathrm{L}} \in \mathrm{M}_{k, k}(\mathbb{C})$, then

$$
\begin{aligned}
A E A^{*} & =\left(\begin{array}{cc}
(-1)^{k} A_{\mathrm{U}} E_{k}+E_{k} A_{\mathrm{U}}^{*} & E_{k} A_{\mathrm{L}}^{*} \\
(-1)^{k} A_{\mathrm{L}} E_{k} & 0
\end{array}\right), \\
B E B^{*} & =\left(\begin{array}{cc}
0 & (-1)^{k+1} B_{\mathrm{U}} E_{k} \\
-E_{k} B_{\mathrm{U}}^{*} & (-1)^{k+1} B_{\mathrm{L}} E_{k}-E_{k} B_{\mathrm{L}}^{*}
\end{array}\right)
\end{aligned}
$$

since

$$
E=\left(\begin{array}{cc}
0 & E_{k} \\
(-1)^{k} E_{k} & 0
\end{array}\right)
$$

with $E_{k}$ having its clear meaning. Note that $E_{k}^{-1}=(-1)^{k+1} E_{k}=E_{k}^{*}$. So, in $\mathcal{N}_{0}^{\mathbb{C}}$, the selfadjointness condition is equivalent to that

$$
A_{\mathrm{U}} E_{k} \text { and } B_{\mathrm{L}} E_{k} \text { are Hermitian, } \quad B_{\mathrm{U}}=E_{k} A_{\mathrm{L}}^{*} E_{k}
$$

For each subset $N \subset \mathbb{N}_{2 n}$ with exactly $n$ elements, set

$$
\mathcal{O}_{N}^{\mathbb{C}}=\mathcal{N}_{N}^{\mathbb{C}} \cap \mathscr{B}^{\mathbb{C}}
$$


Let $\mathrm{H}_{k}$ be the space of $k \times k$ Hermitian complex matrices. Then,

$$
\mathcal{O}_{0}^{\mathbb{C}}=\left\{\left[\begin{array}{cccc}
I & C E_{k} & 0 & E_{k} A_{\mathrm{L}}^{*} E_{k} \\
0 & A_{\mathrm{L}} & -I & D E_{k}
\end{array}\right] ; C, D \in \mathrm{H}_{k}, A_{\mathrm{L}} \in \mathrm{M}_{k, k}(\mathbb{C})\right\} \simeq \mathbb{R}^{4 k^{2}}
$$

and hence is a local coordinate system on $\mathscr{B}^{\mathbb{C}}$. For each preferred subset $N$ of $\mathbb{N}_{2 n}$, we have similar conclusions about $0_{N}^{\mathbb{C}}$. For any two preferred subsets $M$ and $N$ of $\mathbb{N}_{2 n}$, $\mathcal{O}_{M}^{\mathbb{C}} \cap \mathbb{O}_{N}^{\mathbb{C}} \neq \varnothing$, and the transformations between them are rational. In conclusion, we have proved the following results.

THEOREM 3.11. The space $\mathscr{B}^{\mathbb{C}}$ of $2 k$ th-order complex selfadjoint boundary conditions is a connected, compact, and real-analytic $4 k^{2}$-dimensional real submanifold of $\mathbb{A}^{\mathbb{C}}$. It has an atlas of $2^{2 k}$ local coordinate systems with rational transformations among them.

REMARK 3.12. The space of selfadjoint extensions of the minimal operator corresponding to the QDE (2.6) is parameterized by the unitary matrices in dimension $2 k$ and has a dimension of $4 k^{2}-1$, see [18]. Thus, generically, a selfadjoint extension is represented by a 1-parameter family of selfadjoint BCs.

If $N$ is a preferred subset of $\mathbb{N}_{2 n}$, then $O_{N}^{\mathbb{C}}$ is called a canonical coordinate system on $\mathscr{B}^{\mathbb{C}}$. As illustrations, we give the elements in the typical canonical coordinate system $\mathbb{O}_{\mathbb{C}}^{\mathbb{C}}$ for the first two even values of $n$ :

$$
\left[\begin{array}{cccc}
1 & a_{12} & 0 & \overline{a_{22}} \\
0 & a_{22} & -1 & b_{22}
\end{array}\right]
$$

with $a_{12}, b_{22} \in \mathbb{R}$ and $a_{22} \in \mathbb{C}$ if $n=2$, and

$$
\left[\begin{array}{cccccccc}
1 & 0 & a_{13} & a_{14} & 0 & 0 & -\overline{a_{44}} & \overline{a_{34}} \\
0 & 1 & a_{23} & -\overline{a_{13}} & 0 & 0 & \overline{a_{43}} & -\overline{a_{33}} \\
0 & 0 & a_{33} & a_{34} & -1 & 0 & b_{33} & b_{34} \\
0 & 0 & a_{43} & a_{44} & 0 & -1 & b_{43} & -\overline{b_{33}}
\end{array}\right]
$$

with $a_{13}, a_{33}, a_{34}, a_{43}, a_{44}, b_{33} \in \mathbb{C}$ and $a_{14}, a_{23}, b_{34}, b_{43} \in \mathbb{R}$ if $n=4$. In general, $C \in$ $\mathrm{M}_{k, k}(\mathbb{C})$ makes $C E_{k} \in \mathrm{H}_{k}$ if and only if

$$
c_{i, j}=(-1)^{i-j+k+1} \overline{C_{k+1-j, k+1-i}} \quad \forall i, j \in \mathbb{N}_{k},
$$

that is, if and only if $C$ has the form

$$
\left(\begin{array}{cccc}
c_{1,1} & \cdots & c_{1, k-1} & c_{1, k} \\
\vdots & \therefore & c_{2, k-1} & -\overline{c_{1, k-1}} \\
c_{k-1,1} & \therefore & . & \vdots \\
c_{k, 1} & -\overline{c_{k-1,1}} & \cdots & (-1)^{k-1} \overline{c_{1,1}}
\end{array}\right)
$$


while $E_{k} A_{\mathrm{L}}^{*} E_{k}$ is given by

$$
\left(\begin{array}{cccc}
(-1)^{k+1} \overline{a_{n, n}} & \cdots & (-1)^{3} \overline{a_{k+2, n}} & (-1)^{2} \overline{a_{k+1, n}} \\
\vdots & \cdots & \vdots & \vdots \\
(-1)^{2 k-1} \overline{a_{n, k+2}} & \cdots & (-1)^{k+1} \overline{a_{k+2, k+2}} & (-1)^{k} \overline{a_{k+1, k+2}} \\
(-1)^{2 k} \overline{a_{n, k+1}} & \cdots & (-1)^{k+2} \overline{a_{k+2, k+1}} & (-1)^{k+1} \overline{a_{k+1, k+1}}
\end{array}\right) .
$$

As a consequence of our analysis for Theorem 3.11, we have the following fact which is a refinement of Lemma 3.9.

COROLLARY 3.13. For any $2 k$ th-order complex selfadjoint boundary condition $[A \mid B]$, $\operatorname{rank} A=\operatorname{rank} B \geq k$.

Proof. By Lemma 3.8, we can assume that $[A \mid B] \in \mathcal{O}_{\rrbracket}^{\mathbb{C}}$. Using (3.17), from (3.20) we obtain that $\operatorname{rank} A_{\mathrm{L}}=\operatorname{rank} B_{\mathrm{U}}^{*}=\operatorname{rank} B_{\mathrm{U}}$, so $\operatorname{rank} A=k+\operatorname{rank} A_{\mathrm{L}}=k+\operatorname{rank} B_{\mathrm{U}}=\operatorname{rank} B$.

In a linear system representing a BC, usually there are equations involving only $Y(a)$, equations involving only $Y(b)$, and equations involving both $Y(a)$ and $Y(b)$. They will be called equations at $a$, equations at $b$, and coupled equations, respectively. In order to reach the minimum number of coupled equations, we need to apply row operations to the system so that the nonzero rows of the $Y(a)$-coefficient matrix and of the $Y(b)$ coefficient matrix are linearly independent and the two sets of rows have the minimum overlap. In the form given by (3.22), we need to apply row operations to the last $k$ rows of the matrix $(A \mid B)$ so that the nonzero rows of $A_{\mathrm{L}}$ are linearly independent (and at the same time giving up the form of the $I$ block in $A$ ), and then to the first $k$ rows of $(A \mid B)$ so that the nonzero rows of $E_{k} A_{\mathrm{L}}^{*} E_{k}$ are linearly independent (and meanwhile loosing the form of the $-I$ block in $B$ ). For example, the minimum number of coupled equations in the selfadjoint BC

$$
\left[\begin{array}{cccccccc}
1 & 0 & 0 & 0 & 0 & 0 & 0 & 0 \\
0 & 1 & 0 & 0 & 0 & 0 & 1 & -1 \\
0 & 0 & 1 & 0 & -1 & 0 & 0 & 0 \\
0 & 0 & 1 & 0 & 0 & -1 & 0 & 0
\end{array}\right]=\left[\begin{array}{cccccccc}
1 & 0 & 0 & 0 & 0 & 0 & 0 & 0 \\
0 & 1 & 0 & 0 & 0 & 0 & 1 & -1 \\
0 & 0 & 1 & 0 & -1 & 0 & 0 & 0 \\
0 & 0 & 0 & 0 & 1 & -1 & 0 & 0
\end{array}\right]
$$

is 2. Thus, by Lemma 3.8 and the fact that $\operatorname{rank} A_{\mathrm{L}}=\operatorname{rank}\left(E_{k} A_{\mathrm{L}}^{*} E_{k}\right)$, we have proven the following results, special cases of which have appeared in $[5,6]$.

COROLLARY 3.14. The minimum number of coupled equations in a complex selfadjoint boundary condition of an even order is always even. In any form of the boundary condition achieving this minimum number, the number of equations at the left-end point is equal to the number of equations at the right-end point. Moreover, for any even integer $m$ satisfying $0 \leq m \leq 2 k$, there are $2 k$ th-order complex selfadjoint boundary conditions whose minimum numbers of coupled equations are $m$.

By Lemma 3.8 and (3.22), the space $\mathscr{B}_{2 k}^{\mathbb{C}, \mathrm{s}}$ of $2 k$ th-order complex separated selfadjoint BCs is a connected, compact, and real-analytic $2 k^{2}$-dimensional real submanifold of $\mathscr{B}^{\mathbb{C}}$. 
It has an atlas of $2^{2 k}$ local coordinate systems with rational transformations among them. The canonical coordinate systems on $\mathscr{B}_{2 k}^{\mathbb{C}, s}$ are $\mathcal{O}_{N}^{\mathbb{C}, \mathrm{s}}=\mathscr{B}_{2 k}^{\mathbb{C}, \mathrm{s}} \cap \mathcal{O}_{N}^{\mathbb{C}}$, where $N$ varies over the set of preferred subsets of $\mathbb{N}_{2 n}$.

All the above arguments can be restricted to the space $\mathscr{A}^{\mathbb{R}}$ to obtain results about the real selfadjoint BCs. Here we first state the following theorem.

THEOREM 3.15. The space $\mathscr{B}^{\mathbb{R}}$ of $2 k$ th-order real selfadjoint boundary conditions is a connected, compact, and real-analytic $\left(2 k^{2}+k\right)$-dimensional submanifold of $\mathbb{A}^{\mathbb{R}}$. It has an atlas of $2^{2 k}$ local coordinate systems with rational transformations among them.

As in the complex case, the canonical coordinate systems on $\mathscr{B}^{\mathbb{R}}$ are $0_{N}^{\mathbb{R}}=\mathscr{B}^{\mathbb{R}} \cap \mathcal{N}_{N}^{\mathbb{R}}$, where $N$ varies over the set of preferred subsets of $\mathbb{N}_{2 n}$.

The only other thing that we mention is the space $\mathscr{B}_{2 k}^{\mathbb{R}, \mathrm{s}}$ of $2 k$ th-order real separated selfadjoint BCs. By Lemma 3.8 and (3.22), $\mathscr{B}_{2 k}^{\mathbb{R}, \mathrm{s}}$ is a connected, compact, and real-analytic $\left(k^{2}+k\right)$-dimensional submanifold of $\mathscr{B}^{\mathbb{R}}$. It has an atlas of $2^{2 k}$ local coordinate systems with rational transformations among them. The canonical coordinate systems on $\mathscr{B}_{2 k}^{\mathbb{R}, \mathrm{s}}$ are $\mathcal{O}_{N}^{\mathbb{R}, \mathrm{s}}=\mathscr{B}_{2 k}^{\mathbb{R}, \mathrm{s}} \cap \mathrm{O}_{N}^{\mathbb{R}}$, where $N$ varies over the set of preferred subsets of $\mathbb{N}_{2 n}$.

SELFADJOINT BOUNDARY CONDITIONS OF AN ODD ORDER. Let $n=2 k+1$ with $k \in$ $\mathbb{N}$. Now, for any $n$ th-order complex selfadjoint BC $[A \mid B]$, each of $A$ and $B$ has a middle column-its $(k+1)$ th column which does not belong to any symmetric pair.

As in the even-order case, first we have the following two lemmas on the ranks of the left- and right-half blocks in a selfadjoint BC and normalizations of selfadjoint BCs.

LEMmA 3.16. For any $(2 k+1)$ th-order $[A \mid B] \in \mathscr{B}^{\mathbb{C}}, \operatorname{rank} A \geq k+1$ and $\operatorname{rank} B \geq k+1$. Therefore, there is no separated selfadjoint boundary condition of an odd order.

Proof. Here we only show that $\operatorname{rank} A \geq k+1$, while similar arguments give that $\operatorname{rank} B \geq k+1$.

Let $l=\operatorname{rank} A$, then $l \geq 1$. To reach a contradiction, we assume that $l \leq k$. We can assume further that the last $n-l$ rows of $A$ are 0 . Hence, (3.10) holds for some $l \times l$ matrix $C$, and the last $n-l$ rows of $B$ are linearly independent.

If $b_{i, k+1}=0$ for $i=l+1, l+2, \ldots, n$, then a normalization of $B$ as that in the proof of Lemma 3.9 implies a contradiction, since the middle column of $B$ cannot be normalized and $n-l \geq k+1$. Thus, we must have that $b_{i, k+1} \neq 0$ for some integer $i$ satisfying $l+1 \leq i \leq n$.

Now, normalize $B$ such that its middle column is normalized (first), and the number $n_{B}$ of symmetric pairs of unnormalized columns is minimum for such a normalization. By part of the arguments in the proof of Lemma 3.9 (putting the nonzero entries of the symmetric pair of normalized columns used into the right rows), we must have that ( $l=k$ and) $n_{B}=0$. We can assume that $b_{n, k+1}=-1$. Then, the $(n, n)$-entry of $B E B^{*}$ equals $(-1)^{k+1}$, still contradicting (3.10). Therefore, we have shown that rank $A \geq k+1$.

LEMMA 3.17. Every odd-order $[A \mid B] \in \mathscr{B}^{\mathbb{C}}$ has a normalization such that the middle column of $A$ is normalized and each symmetric pair of columns in A or in B has one (and hence only one) normalized column. 
Proof. If the middle column of $A$ is zero, we normalize $k$ columns of $A$ such that the number of symmetric pairs of unnormalized columns is minimum. Then,

$$
A E A^{*}=\left(\begin{array}{cc}
C & D \\
H & 0
\end{array}\right)
$$

for some $k \times k$ matrix $C, k \times(k+1)$ matrix $D$, and $(k+1) \times k$ matrix $H$. If $b_{i, k+1}=0$ for $i=k+1, k+2, \ldots, n$, then we normalize $k+1$ columns of $B$ such that the number of symmetric pairs of unnormalized columns is minimum, and obtain a nonzero entry in the lower right $(k+1) \times(k+1)$ block of $B E B^{*}$, which is impossible. So, we can assume that $b_{n, k+1} \neq 0$. Then, we normalize $k+1$ columns of $B$ with its middle column normalized, and the number of symmetric pairs of unnormalized columns is minimum for such a normalization. We can assume further that $b_{n, k+1}=-1$ and the minimum number is 0 . Then, the $(n, n)$-entry of $B E B^{*}$ is $(-1)^{k+1}$, which is impossible. Therefore, the middle column of $A$ is nonzero.

Normalize $k+1$ columns of $A$ such that its middle column is normalized and the number $n_{A}$ of symmetric pairs of unnormalized columns of $A$ is minimum for such a normalization. Then,

$$
A E A^{*}=\left(\begin{array}{ccc}
C & \hat{D} & D \\
\hat{H} & d & 0 \\
H & 0 & 0
\end{array}\right)=B E B^{*}
$$

for some $k \times k$ matrices $C, D$, and $H, k \times 1$ matrix $\hat{D}, 1 \times k$ matrix $\hat{H}$, and constant $d$. Moreover, the last row of $A E A^{*}$ is just a rearrangement of the last row of $A$, with some entries signs changed. As in the even-order case, the last $k$ rows of $B$ are then linearly independent.

If the rank of

$$
\left(\begin{array}{cccccc}
b_{k+2,1} & \ldots & b_{k+2, k} & b_{k+2, k+2} & \ldots & b_{k+2, n} \\
\vdots & \vdots & \vdots & \vdots & \vdots & \vdots \\
b_{n, 1} & \ldots & b_{n, k} & b_{n, k+2} & \ldots & b_{n, n}
\end{array}\right)
$$

is less than $k$, then we can assume that

$$
b_{n, 1}=\cdots=b_{n, k}=b_{n, k+2}=\cdots=b_{n, n}=0 .
$$

Thus, $b_{n, k+1} \neq 0$, and hence the $(n, n)$-entry of $B E B^{*}$ is

$$
(-1)^{k+1} b_{n, k+1} \overline{b_{n, k+1}} \neq 0,
$$

contradicting (3.30) above. Therefore, we can always normalize $k$ columns of $B$ such that its middle column is unnormalized and the number $n_{B}$ of symmetric pairs of unnormalized columns is minimum for such a normalization.

If $n_{B}>0$, then $k \geq 2$, and there is an $m \in \mathbb{N}_{k}$ such that both $B$ 's $m$ th column and its $(n+1-m)$ th column are normalized. We can assume that

$$
b_{2 k, m}=b_{n, n+1-m}=-1 \text {. }
$$


Thus, the lower right $2 \times 2$ block of $B E B^{*}$ is

$$
\left(\begin{array}{cc}
(-1)^{k+1} b_{2 k, k+1} \overline{b_{2 k, k+1}} & (-1)^{k+1} b_{2 k, k+1} \overline{b_{n, k+1}}+(-1)^{m} \\
(-1)^{m}+(-1)^{k+1} b_{n, k+1} \overline{b_{2 k, k+1}} & (-1)^{k+1} b_{n, k+1} \overline{b_{n, k+1}}
\end{array}\right) \neq 0,
$$

contradicting (3.30). Therefore, $n_{B}=0$, and

$$
B E B^{*}=\left(\begin{array}{cc}
0 & K \\
L & M
\end{array}\right)+(-1)^{k+1}\left(b_{i, k+1} \overline{b_{j, k+1}}\right)_{n \times n}
$$

for some $(k+1) \times k$ matrix $K, k \times(k+1)$ matrix $L$, and $k \times k$ matrix $M$.

Then, by some arguments similar to those in the previous paragraph, we must also have $n_{A}=0$.

As in the even-order case, a subset $N \subset \mathbb{N}_{2 n}$ with exactly $n$ elements is called a preferred subset if $k+1 \in N$; and for any $i \in \mathbb{N}_{k}$, exactly one of $i$ and $n+1-i$ is in $N$ and exactly one of $n+i$ and $2 n+1-i$ is in $N$. If $N$ is a preferred subset of $\mathbb{N}_{2 n}$, then the canonical coordinate system $\mathcal{N}_{N}^{\mathbb{C}}$ on $\mathscr{A}^{\mathbb{C}}$ is called a preferred coordinate system. For example, when $n=3, \mathcal{N}_{1,2,4}^{\mathbb{C}}, \mathcal{N}_{1,2,6}^{\mathbb{C}}, \mathcal{N}_{2,3,4}^{\mathbb{C}}$, and $\mathcal{N}_{2,3,6}^{\mathbb{C}}$ are preferred, while $\mathcal{N}_{1,2,3}^{\mathbb{C}}, \mathcal{N}_{1,2,5}^{\mathbb{C}}$, $\mathcal{N}_{1,4,5}^{\mathbb{C}}, \mathcal{N}_{1,5,6}^{\mathbb{C}}, \mathcal{N}_{3,4,5}^{\mathbb{C}}, \mathcal{N}_{3,5,6}^{\mathbb{C}}$, and $\mathcal{N}_{4,5,6}^{\mathbb{C}}$ are not. With this new definition, Lemma 3.17 just says that every odd-order selfadjoint $B C$ is in a preferred coordinate system on $A^{\mathbb{C}}$. By Lemma 3.8, solving the selfadjointness equation $A E A^{*}=B E B^{*}$ in any preferred coordinate system on $\mathscr{A}^{\mathbb{C}}$ is equivalent to solving it in $\mathcal{N}_{\mathbb{J}}^{\mathbb{C}}$, where

$$
\mathbb{J}=\{1,2, \ldots, k+1, n+1, n+2, \ldots, 3 k+1\} .
$$

If we set

$$
A=\left(\begin{array}{ccc}
I & 0 & A_{\mathrm{U}} \\
0 & 1 & A_{\mathrm{M}} \\
0 & 0 & A_{\mathrm{L}}
\end{array}\right), \quad B=\left(\begin{array}{ccc}
0 & b_{\mathrm{U}} & B_{\mathrm{U}} \\
0 & b_{\mathrm{M}} & B_{\mathrm{M}} \\
-I & b_{\mathrm{L}} & B_{\mathrm{L}}
\end{array}\right)
$$

where $A_{\mathrm{U}}, A_{\mathrm{L}}, B_{\mathrm{U}}, B_{\mathrm{L}} \in \mathrm{M}_{k, k}(\mathbb{C}), b_{\mathrm{U}}, b_{\mathrm{L}} \in \mathrm{M}_{k, 1}(\mathbb{C}), A_{\mathrm{M}}, B_{\mathrm{M}} \in \mathrm{M}_{1, k}(\mathbb{C})$, and $b_{\mathrm{M}}$ is a constant, then

$$
\begin{aligned}
A E A^{*}= & \left(\begin{array}{ccc}
(-1)^{k+1} A_{\mathrm{U}} E_{k}+E_{k} A_{\mathrm{U}}^{*} & E_{k} A_{\mathrm{M}}^{*} & E_{k} A_{\mathrm{L}}^{*} \\
(-1)^{k+1} A_{\mathrm{M}} E_{k} & (-1)^{k+1} & 0 \\
(-1)^{k+1} A_{\mathrm{L}} E_{k} & 0 & 0
\end{array}\right), \\
B E B^{*}= & \left(\begin{array}{ccc}
0 & 0 & (-1)^{k} B_{\mathrm{U}} E_{k} \\
0 & 0 & (-1)^{k} B_{\mathrm{M}} E_{k} \\
-E_{k} B_{\mathrm{U}}^{*} & -E_{k} B_{\mathrm{M}}^{*} & (-1)^{k} B_{\mathrm{L}} E_{k}-E_{k} B_{\mathrm{L}}^{*}
\end{array}\right) \\
& +(-1)^{k+1}\left(\begin{array}{ccc}
b_{\mathrm{U}} b_{\mathrm{U}}^{*} & b_{\mathrm{U}} \overline{b_{\mathrm{M}}} & b_{\mathrm{U}} b_{\mathrm{L}}^{*} \\
b_{\mathrm{M}} b_{\mathrm{U}}^{*} & b_{\mathrm{M}} \overline{b_{\mathrm{M}}} & b_{\mathrm{M}} b_{\mathrm{L}}^{*} \\
b_{\mathrm{L}} b_{\mathrm{U}}^{*} & b_{\mathrm{L}} \overline{b_{\mathrm{M}}} & b_{\mathrm{L}} b_{\mathrm{L}}^{*}
\end{array}\right),
\end{aligned}
$$


since

$$
E=\left(\begin{array}{ccc}
0 & 0 & E_{k} \\
0 & (-1)^{k+1} & 0 \\
(-1)^{k+1} E_{k} & 0 & 0
\end{array}\right)
$$

So, $A E A^{*}=B E B^{*}$ can be rewritten as

$$
\begin{aligned}
& A_{\mathrm{U}} E_{k}+\left(A_{\mathrm{U}} E_{k}\right)^{*}=b_{\mathrm{U}} b_{\mathrm{U}}^{*}, \quad A_{\mathrm{M}}=(-1)^{k+1} b_{\mathrm{M}} b_{\mathrm{U}}^{*} E_{k}, \\
& B_{\mathrm{U}}=(-1)^{k+1} b_{\mathrm{U}} b_{\mathrm{L}}^{*} E_{k}-E_{k} A_{\mathrm{L}}^{*} E_{k}, \quad b_{\mathrm{M}} \in \mathbb{S}^{1}, \\
& B_{\mathrm{M}}=(-1)^{k+1} b_{\mathrm{M}} b_{\mathrm{L}}^{*} E_{k}, \quad B_{\mathrm{L}} E_{k}+\left(B_{\mathrm{L}} E_{k}\right)^{*}=b_{\mathrm{L}} b_{\mathrm{L}}^{*} .
\end{aligned}
$$

In particular, both $A_{\mathrm{U}} E_{k}-(1 / 2) b_{\mathrm{U}} b_{\mathrm{U}}^{*}$ and $B_{\mathrm{L}} E_{k}-1 / 2 b_{\mathrm{L}} b_{\mathrm{L}}^{*}$ are skew-Hermitian matrices. For each subset $N$ of $\mathbb{N}_{2 n}$ with exactly $n$ elements, set

$$
\mathcal{O}_{N}^{\mathbb{C}}=\mathcal{N}_{N}^{\mathbb{C}} \cap \mathscr{B}^{\mathbb{C}}
$$

Let $\mathrm{W}_{k}$ be the space of $k \times k$ skew-Hermitian complex matrices. Then, the elements of $\mathrm{O}_{\rrbracket}^{\mathbb{C}}$ are given by

$$
\left[\begin{array}{cccccc}
I & 0 & C E_{k}+\frac{(-1)^{k+1}}{2} b_{\mathrm{U}} b_{\mathrm{U}}^{*} E_{k} & 0 & b_{\mathrm{U}} & (-1)^{k+1} b_{\mathrm{U}} b_{\mathrm{L}}^{*} E_{k}-E_{k} A_{\mathrm{L}}^{*} E_{k} \\
0 & 1 & (-1)^{k+1} b_{\mathrm{M}} b_{\mathrm{U}}^{*} E_{k} & 0 & b_{\mathrm{M}} & (-1)^{k+1} b_{\mathrm{M}} b_{\mathrm{L}}^{*} E_{k} \\
0 & 0 & A_{\mathrm{L}} & -I & b_{\mathrm{L}} & D E_{k}+\frac{(-1)^{k+1}}{2} b_{\mathrm{L}} b_{\mathrm{L}}^{*} E_{k}
\end{array}\right]
$$

with $C, D \in \mathrm{W}_{k}, A_{\mathrm{L}} \in \mathrm{M}_{k, k}(\mathbb{C}), b_{\mathrm{U}}, b_{\mathrm{L}} \in \mathrm{M}_{k, 1}(\mathbb{C})$, and $b_{\mathrm{M}} \in \mathbb{S}^{1}$. Thus,

$$
\mathbb{O}_{\mathbb{D}}^{\mathbb{C}} \simeq \mathbb{R}^{4 k^{2}+4 k} \times \mathbb{S}^{1}
$$

and hence is a local cylindrical coordinate system on $\Re^{\mathbb{C}}$. For each preferred subset $N$ of $\mathbb{N}_{2 n}$, we have similar conclusions about $\mathbb{O}_{N}^{\mathbb{C}}$. For any two preferred subsets $M$ and $N$ of $\mathbb{N}_{2 n}, \mathcal{O}_{M}^{\mathbb{C}} \cap \mathcal{O}_{N}^{\mathbb{C}} \neq \varnothing$, and the transformations between them are rational. In conclusion, we have proved the following results.

THEOREM 3.18. The space $\mathscr{B}^{\mathbb{C}}$ of $(2 k+1)$ th-order complex selfadjoint boundary conditions is a connected, compact, and real-analytic $(2 k+1)^{2}$-dimensional real submanifold of $\mathbb{A}^{\mathbb{C}}$. It has an atlas of $2^{2 k}$ local cylindrical coordinate systems with rational transformations among them.

There is also a remark in the odd-order case similar to Remark 3.12 in the even-order case. We omit the details. 
If $N$ is a preferred subset of $\mathbb{N}_{2 n}$, then $O_{N}^{\mathbb{C}}$ is called a canonical cylindrical coordinate system on $\mathscr{B}_{\mathbb{C}}^{\mathbb{C}}$. As illustrations, we give the elements in the typical canonical cylindrical coordinate system $O_{\S}^{\mathbb{C}}$ for the first two odd values of $n$ :

$$
\left[\begin{array}{cccccc}
1 & 0 & -\frac{1}{2} b_{1} \overline{b_{1}}+i r & 0 & b_{1} & -\overline{a_{33}}-b_{1} \overline{b_{3}} \\
0 & 1 & -\overline{b_{1}} b_{2} & 0 & b_{2} & -b_{2} \overline{b_{3}} \\
0 & 0 & a_{33} & -1 & b_{3} & -\frac{1}{2} b_{3} \overline{b_{3}}+i s
\end{array}\right]
$$

with $r, s \in \mathbb{R}, a_{33}, b_{1}, b_{3} \in \mathbb{C}$, and $b_{2} \in \mathbb{S}^{1}$ if $n=3$, and

$$
\left[\begin{array}{cccccccccc}
1 & 0 & 0 & a_{14} & \frac{1}{2} b_{1} \overline{b_{1}}+i r & 0 & 0 & b_{1} & \overline{a_{55}}-b_{1} \overline{b_{5}} & -\overline{a_{45}}+b_{1} \overline{b_{4}} \\
0 & 1 & 0 & \frac{-1}{2} b_{2} \overline{b_{2}}+i s & \overline{a_{14}}+b_{2} \overline{b_{1}} & 0 & 0 & b_{2} & -\overline{a_{54}}-b_{2} \overline{b_{5}} & \overline{a_{44}}+b_{2} \overline{b_{4}} \\
0 & 0 & 1 & -\overline{b_{2}} b_{3} & \overline{b_{1}} b_{3} & 0 & 0 & b_{3} & -b_{3} \overline{b_{5}} & b_{3} \overline{b_{4}} \\
0 & 0 & 0 & a_{44} & a_{45} & -1 & 0 & b_{4} & b_{44} & \frac{1}{2} b_{4} \overline{b_{4}}+i x \\
0 & 0 & 0 & a_{54} & a_{55} & 0 & -1 & b_{5} & \frac{-1}{2} b_{5} \overline{b_{5}}+i y & \overline{b_{44}}+b_{5} \overline{b_{4}}
\end{array}\right]
$$

with $a_{14}, a_{44}, a_{45}, a_{54}, a_{55}, b_{1}, b_{2}, b_{4}, b_{44}, b_{5} \in \mathbb{C}, r, s, x, y \in \mathbb{R}$, and $b_{3} \in \mathbb{S}^{1}$ if $n=5$. In general, given $\mathbf{v}=\left(\begin{array}{llll}v_{1} & v_{2} & \cdots & v_{k}\end{array}\right)^{t} \in \mathrm{M}_{k, 1}(\mathbb{C})$, a $k \times k$ complex matrix $C$ makes $C E_{k}-$ $(1 / 2) \mathbf{v} \mathbf{v}^{*} \in \mathrm{W}_{k}$ if and only if

$$
c_{i, j}=(-1)^{i-j+k} \overline{c_{k+1-j, k+1-i}}+(-1)^{j} v_{i} \overline{v_{k+1-j}} \quad \forall i, j \in \mathbb{N}_{k},
$$

that is, if and only if $C$ has the form

$$
\left(\begin{array}{cccc}
c_{1,1} & \ldots & c_{1, k-1} & \frac{(-1)^{k}}{2} v_{1} \overline{v_{1}}+i r_{1} \\
\vdots & \therefore & \frac{(-1)^{k-1}}{2} v_{2} \overline{v_{2}}+i r_{2} & \overline{c_{1, k-1}}+(-1)^{k} v_{2} \overline{v_{1}} \\
c_{k-1,1} & \therefore & \therefore & \vdots \\
\frac{-1}{2} v_{k} \overline{v_{k}}+i r_{k} & \overline{c_{k-1,1}}+(-1)^{2} v_{k} \overline{v_{k-1}} & \cdots & (-1)^{k} \overline{c_{1,1}}+(-1)^{k} v_{k} \overline{v_{1}}
\end{array}\right)
$$

with $r_{1}, r_{2}, \ldots, r_{k} \in \mathbb{R}$, while

$$
(-1)^{k+1} \mathbf{v}^{*} E_{k}=\left(\begin{array}{llll}
-\overline{v_{k}} & \cdots & (-1)^{k-1} \overline{v_{2}} & (-1)^{k} \overline{v_{1}}
\end{array}\right)
$$


and $(-1)^{k+1} b_{\mathrm{U}} b_{\mathrm{L}}^{*} E_{k}-E_{k} A_{\mathrm{L}}^{*} E_{k}$ is given by

$$
\begin{gathered}
\left(\begin{array}{cccc}
(-1)^{k} \overline{a_{n, n}} & \cdots & (-1)^{2} \overline{a_{k+3, n}} & -\overline{a_{k+2, n}} \\
(-1)^{k+1} \overline{a_{n, 2 k}} & \cdots & (-1)^{3} \overline{a_{k+3,2 k}} & (-1)^{2} \overline{a_{k+2,2 k}} \\
\vdots & \cdots & \vdots & \vdots \\
(-1)^{2 k-1} \overline{a_{n, k+2}} & \cdots & (-1)^{k+1} \overline{a_{k+3, k+2}} & (-1)^{k} \overline{a_{k+2, k+2}}
\end{array}\right) \\
+\left(\begin{array}{cccc}
-b_{1} \overline{b_{n}} & \cdots & (-1)^{k-1} b_{1} \overline{b_{k+3}} & (-1)^{k} b_{1} \overline{b_{k+2}} \\
-b_{2} \overline{b_{n}} & \cdots & (-1)^{k-1} b_{2} \overline{b_{k+3}} & (-1)^{k} b_{2} \overline{b_{k+2}} \\
\vdots & \cdots & \vdots & \vdots \\
-b_{k} \overline{b_{n}} & \cdots & (-1)^{k-1} b_{k} \overline{b_{k+3}} & (-1)^{k} b_{k} \overline{b_{k+2}}
\end{array}\right),
\end{gathered}
$$

where we have abbreviated the middle column of $B$ as $\left(b_{1} b_{2} \cdots b_{n}\right)^{t}$.

As a consequence of our analysis for Theorem 3.18, we have the following fact which is a refinement of Lemma 3.16.

COROLLARY 3.19. For any $(2 k+1)$ th-order complex selfadjoint boundary condition $[A \mid B], \operatorname{rank} A=\operatorname{rank} B \geq k+1$.

Proof. By Lemma 3.8, we can assume that $[A \mid B] \in 0_{\S}^{\mathbb{C}}$. Since $b_{\mathrm{M}} \neq 0$ by (3.42), we can apply only row operations to $[A \mid B]$ such that $b_{U}=0$. Then, $[A \mid B]$ has the form

$$
\left[\begin{array}{cccccc}
I & a_{\mathrm{U}} & A_{\mathrm{U}} & 0 & 0 & B_{\mathrm{U}} \\
0 & 1 & A_{\mathrm{M}} & 0 & b_{\mathrm{M}} & B_{\mathrm{M}} \\
0 & 0 & A_{\mathrm{L}} & -I & b_{\mathrm{L}} & B_{\mathrm{L}}
\end{array}\right]
$$

where $a_{\mathrm{U}}$ is a $k \times 1$ matrix. Now, $A E A^{*}=B E B^{*}$ implies that $E_{k} A_{\mathrm{L}}^{*}=(-1)^{k} B_{\mathrm{U}} E_{k}$. So,

$$
\operatorname{rank} A_{\mathrm{L}}=\operatorname{rank} B_{\mathrm{U}},
$$

and $\operatorname{rank} A=k+1+\operatorname{rank} A_{\mathrm{L}}=k+1+\operatorname{rank} B_{\mathrm{U}}=\operatorname{rank} B$.

By Lemma 3.8, (3.53), (3.54), and the discussions right before Corollary 3.14, we have the following results that are similar to those in Corollary 3.14.

COROLLARY 3.20. The minimum number of coupled equations in a complex selfadjoint boundary condition of an odd order is always odd. In any form of the boundary condition achieving this minimum number, the number of equations at the left-end point is equal to the number of equations at the right-end point. Moreover, for any odd integer $m$ satisfying $1 \leq m \leq 2 k+1$, there are $(2 k+1)$ th-order complex selfadjoint boundary conditions whose minimum numbers of coupled equations are $m$.

Finally, we restrict our attention to the space $\mathscr{B}^{\mathbb{R}}$ of real selfadjoint BCs of the odd order $n$. Let $N$ be a preferred subset of $\mathbb{N}_{2 n}$. By Lemma 3.8 and (3.42), if $[A \mid B] \in \mathcal{O}_{N}^{\mathbb{C}} \cap \mathscr{B}^{\mathbb{R}}$ has the normalized form corresponding to (3.45), then ( $A, B$ are real, and) $b_{k+1, k+1}= \pm 1$. 
Thus,

$\mathcal{O}_{N, \pm}^{\mathbb{R}}=:\left\{[A \mid B] \in \mathcal{O}_{N}^{\mathbb{C}} \cap \mathscr{B}^{\mathbb{R}} ; b_{k+1, k+1}= \pm 1\right.$ when $[A \mid B]$ is normalized $\} \simeq \mathbb{R}^{2 k^{2}+k}$

are local coordinate systems on $\mathscr{B}^{\mathbb{R}}$. There are $2^{2 k+1}$ such local coordinate systems on $\mathscr{B}^{\mathbb{R}}$, to be called the canonical coordinate systems on $\mathscr{B}^{\mathbb{R}}$.

THEOREM 3.21. The space $\mathscr{B}^{\mathbb{R}}$ of $(2 k+1)$ th-order real selfadjoint boundary conditions is a compact and real-analytic $\left(2 k^{2}+k\right)$-dimensional submanifold of $\mathbb{A}^{\mathbb{R}}$. It has an atlas of $2^{2 k+1}$ local coordinate systems with rational transformations among them and exactly two connected components.

Proof. The only thing left is to determine the number of connected components in $\mathscr{B}^{\mathbb{R}}$. If $[A \mid B] \in \mathrm{O}_{\mathbb{J}, \pm}^{\mathbb{R}}$ is in the form (3.53), then from $E_{k} A_{\mathrm{L}}^{t}=(-1)^{k} B_{\mathrm{U}} E_{k}$ we obtain that

$$
\operatorname{det} A=\operatorname{det} A_{\mathrm{L}}=(-1)^{k} \operatorname{det} B_{\mathrm{U}}=(-1)^{k} b_{\mathrm{M}} \operatorname{det} B .
$$

Hence, on each canonical coordinate system on $\mathscr{B}^{\mathbb{R}}$, either we always have that $\operatorname{det} A=$ $\operatorname{det} B$ or we always have that $\operatorname{det} A=-\operatorname{det} B$. On the other hand, if $[A \mid B] \in \mathscr{B}^{\mathbb{R}}$ has $\operatorname{det} A \neq 0$, then $\operatorname{det} B \neq 0$, and $[A \mid B]$ can be normalized into the form in (3.45), that is, either $[A \mid B] \in \mathbb{O}_{\mathbb{\jmath},+}^{\mathbb{R}}$ or $[A \mid B] \in \mathbb{O}_{\mathfrak{\downarrow},-}^{\mathbb{R}}$, $\operatorname{depending}$ on $\operatorname{det} A=(-1)^{k} \operatorname{det} B$ or $\operatorname{det} A=$ $(-1)^{k+1} \operatorname{det} B$. So, all the canonical coordinate systems always satisfying $\operatorname{det} A=\operatorname{det} B$ overlap, and all the other canonical coordinate systems also overlap. When two canonical coordinate systems overlap, their common part is open and hence must have a $\mathrm{BC}$ $[A \mid B]$ with $\operatorname{det} A \neq 0$ by dimension counting. Thus, on any two overlapping canonical coordinate systems, either we always have that $\operatorname{det} A=\operatorname{det} B$ or we always have that $\operatorname{det} A=-\operatorname{det} B$. So, the canonical coordinate systems always satisfying $\operatorname{det} A=\operatorname{det} B$ form a connected component of $\mathscr{B}^{\mathbb{R}}$, and all the other canonical coordinate systems form another connected component. Therefore, $\mathscr{B}^{\mathbb{R}}$ has exactly two connected components.

We will denote by $\mathscr{R}_{-}^{\mathbb{R}}$ the connected component of $\mathscr{B}^{\mathbb{R}}$ containing $\mathbb{O}_{\downarrow,-}^{\mathbb{R}}$, and $\mathscr{P}_{+}^{\mathbb{R}}$ the other component of $\mathscr{B}^{\mathbb{R}}$.

4. Group actions and $\lambda$-hypersurfaces. In this section, we first discuss some Lie group actions on spaces of BCs. Then, for a given QDE of order $n$, we characterize the set of complex BCs that have a complex number $\lambda$ as an eigenvalue of geometric multiplicity $n$, the set of complex BCs that have $\lambda$ as an eigenvalue, and the set of real $\mathrm{BC}$ that have a real number $\lambda$ as an eigenvalue. The determination of the latter two sets uses the Lie group actions to be discussed. Moreover, we also present a topological description of each of the latter two sets. Finally, we give an example to indicate that the set of real selfadjoint BCs having a real number $\lambda$ as an eigenvalue is complicated in general.

Given

$$
\left(\begin{array}{cc}
G & H \\
K & L
\end{array}\right) \in \mathrm{GL}(2 n, \mathbb{R}),
$$


where $G, H, K, L \in \mathrm{M}_{n, n}(\mathbb{R})$, the well-defined map

$$
[A \mid B] \longmapsto[A G+B K \mid A H+B L]
$$

is a diffeomorphism of $\mathscr{A}^{\mathbb{R}}$ (onto itself). Thus, the group GL(2n, $\left.\mathbb{R}\right)$ acts on $\mathscr{A}^{\mathbb{R}}$ from the right.

For $\mathbb{K}=\mathbb{C}$ or $\mathbb{R}$, consider the real Lie subgroup

$$
\mathbf{G}_{n}^{\mathbb{K}}=\left\{M \in \mathrm{GL}(n, \mathbb{K}) ; M E M^{*}=E\right\}
$$

of $\operatorname{GL}(n, \mathbb{K})$. The Lie algebra of $\mathbf{G}_{n}^{\mathbb{K}}$ is

$$
\mathscr{G}_{n}^{\mathbb{K}}=\left\{M \in \operatorname{gl}(n, \mathbb{K}) ; M E+E M^{*}=0\right\} .
$$

Direct calculations show the following: for $n=2 k$ with $k \in \mathbb{N}$,

$$
\left(\begin{array}{cc}
I & 0 \\
0 & (-1)^{k} E_{k}
\end{array}\right)^{-1} E\left(\begin{array}{cc}
I & 0 \\
0 & (-1)^{k} E_{k}
\end{array}\right)=\left(\begin{array}{cc}
0 & -I_{k} \\
I_{k} & 0
\end{array}\right),
$$

which implies that $\mathbf{G}_{2 k}^{\mathbb{R}}$ is a conjugate of the real symplectic group

$$
\operatorname{Sp}(2 k, \mathbb{R})=\left\{M \in \mathrm{GL}(n, \mathbb{R}) ; M\left(\begin{array}{cc}
0 & -I_{k} \\
I_{k} & 0
\end{array}\right) M^{t}=\left(\begin{array}{cc}
0 & -I_{k} \\
I_{k} & 0
\end{array}\right)\right\},
$$

and $\mathbf{G}_{2 k}^{\mathbb{C}}$ is a conjugate of the symplectic group

$$
\operatorname{Sp}(2 k)=\left\{M \in \operatorname{GL}(n, \mathbb{C}) ; M\left(\begin{array}{cc}
0 & -I_{k} \\
I_{k} & 0
\end{array}\right) M^{*}=\left(\begin{array}{cc}
0 & -I_{k} \\
I_{k} & 0
\end{array}\right)\right\},
$$

while for $n=2 k+1$ with $k \in \mathbb{N}$,

$$
T^{-1} E T=\left(\begin{array}{ccc}
-I_{k} & 0 & 0 \\
0 & (-1)^{k+1} & 0 \\
0 & 0 & I_{k}
\end{array}\right)
$$

where

$$
T=\left(\begin{array}{ccc}
I_{k} & 0 & J_{k} \\
0 & 1 & 0 \\
(-1)^{k} E_{k} & 0 & (-1)^{k+1} D_{k}
\end{array}\right)
$$

with

$$
J_{k}=\left(\begin{array}{cccc}
0 & \cdots & 0 & 1 \\
\vdots & . & 1 & 0 \\
0 & . & . & \vdots \\
1 & 0 & \cdots & 0
\end{array}\right), \quad D_{k}=\left(\begin{array}{cccc}
-1 & 0 & \cdots & 0 \\
0 & (-1)^{2} & \ddots & \vdots \\
\vdots & \ddots & \ddots & 0 \\
0 & \cdots & 0 & (-1)^{k}
\end{array}\right)
$$


which yields that $\mathbf{G}_{2 k+1}^{\mathbb{R}}$ is a conjugate of the special pseudo-orthogonal group

$$
\mathrm{SO}(p, q)=\left\{M \in \mathrm{GL}(n, \mathbb{R}) ; M\left(\begin{array}{cc}
-I_{p} & 0 \\
0 & I_{q}
\end{array}\right) M^{t}=\left(\begin{array}{cc}
-I_{p} & 0 \\
0 & I_{q}
\end{array}\right)\right\},
$$

and $\mathbf{G}_{2 k+1}^{\mathbb{C}}$ is a conjugate of the special pseudo-unitary group

$$
\mathrm{SU}(p, q)=\left\{M \in \mathrm{GL}(n, \mathbb{C}) ; M\left(\begin{array}{cc}
-I_{p} & 0 \\
0 & I_{q}
\end{array}\right) M^{*}=\left(\begin{array}{cc}
-I_{p} & 0 \\
0 & I_{q}
\end{array}\right)\right\},
$$

where $p=k+\left(1+(-1)^{k}\right) / 2$ and $q=k+\left(1-(-1)^{k}\right) / 2$. Therefore, $\mathbf{G}_{n}^{\mathbb{R}}$ and $\mathbf{G}_{n}^{\mathbb{C}}$ are connected. Moreover,

$$
\begin{aligned}
& \mathbf{G}_{2}^{\mathbb{R}}=\operatorname{Sp}(2, \mathbb{R})=\operatorname{SL}(2, \mathbb{R}), \\
& \mathbf{G}_{2}^{\mathbb{C}}=\operatorname{Sp}(2)=\left\{z K ; z \in \mathbb{S}^{1}, K \in \operatorname{SL}(2, \mathbb{R})\right\},
\end{aligned}
$$

which have been used in [15].

The subgroup

$$
\left\{\left(\begin{array}{cc}
G & 0 \\
0 & L
\end{array}\right) ; G, L \in \mathbf{G}_{n}^{\mathbb{R}}\right\}
$$

of $\operatorname{GL}(2 n, \mathbb{R})$ actually acts on $\mathscr{B}^{\mathbb{R}}$ as onto diffeomorphisms, and also on $\mathscr{B}^{\mathbb{R}, \mathrm{s}}$ as onto diffeomorphisms when $n$ is even. Moreover, for any $G \in \mathrm{GL}(n, \mathbb{R})$, the action of

$$
\operatorname{diag}(G, I)=:\left(\begin{array}{cc}
G & 0 \\
0 & I
\end{array}\right)
$$

on $\mathscr{A}^{\mathbb{R}}$ leaves $\mathcal{N}_{N}^{\mathbb{R}}$ invariant, where $N=\llbracket$ if $n$ is even and $N=\rrbracket$ if $n$ is odd, and it also leaves the space $\mathscr{A}^{\mathbb{R}, \mathrm{s}}$ of separated real BCs invariant; and for any $\Psi \in \mathbf{G}_{n}^{\mathbb{R}}$, the action

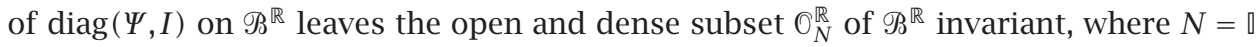
if $n$ is even and $N=\mathbb{J}$ if $n$ is odd. When there is no confusion, the image of a real BC $[A \mid B]$ under the action of $\operatorname{diag}(G, I)$ will be abbreviated as $[A \mid B] \cdot G$, while the image of a subset $\mathscr{Y}$ of $\mathscr{A}^{\mathbb{R}}$ will be written as $\mathscr{Y} . G$. Note that when $n$ is even, $\mathscr{B}^{\mathbb{R}, \mathrm{s}} \cdot G=\mathscr{B}^{\mathbb{R}, \mathrm{s}}$ for any $G \in \mathbf{G}_{n}^{\mathbb{R}}$.

Proposition 4.1. If $n$ is odd, then $\left(\mathscr{B}_{-}^{\mathbb{R}}\right), G=\mathscr{B}_{-}^{\mathbb{R}}$ and $\left(\mathscr{B}_{+}^{\mathbb{R}}\right) . G=\mathscr{B}_{+}^{\mathbb{R}}$ for any $G \in \mathbf{G}_{n}^{\mathbb{R}}$.

Proof. Our claim is true for $G=I$. Fix an arbitrary $G \in \mathbf{G}_{n}^{\mathbb{R}}$. The connectedness of $\mathbf{G}_{n}^{\mathbb{R}}$ and the openness of $\mathscr{R}_{ \pm}^{\mathbb{R}}$ imply that $\left(\mathscr{S}_{ \pm}^{\mathbb{R}}\right) . G \subseteq \mathscr{B}_{ \pm}^{\mathbb{R}}$. Hence, the claim is true for $G$.

Similarly, the Lie group GL $(2 n, \mathbb{C})$ acts on $\mathbb{A}^{\mathbb{C}}$ from the right, the real Lie subgroup

$$
\left\{\operatorname{diag}(G, H) ; G, H \in \mathbf{G}_{n}^{\mathbb{C}}\right\}
$$

of $\operatorname{GL}(2 n, \mathbb{C})$ acts on $\mathscr{B}^{\mathbb{C}}$, and the meanings of $[A \mid B] . G$ and $\mathscr{Y} . G$ in this general situation are obvious. Note that when $n$ is even, $\mathscr{B}^{\mathbb{C}, \mathrm{s}} \cdot G=\mathscr{B}^{\mathbb{C}, \mathrm{s}}$ for any $G \in \mathbf{G}_{n}^{\mathbb{C}}$.

We will always let (2.6) be the given QDE. Recall that its matrix form is (2.9). We have the following results about the values of its fundamental matrix $\Phi$. 
Proposition 4.2. (i) For any $\lambda \in \mathbb{C}$ and $x \in[a, b], \Phi(x, \lambda) \in \operatorname{GL}(n, \mathbb{C})$.

(ii) Assume that $F$ is E-symmetric and $w$ is real-valued, then $\Phi(x, \lambda) \in \mathbf{G}_{n}^{\mathbb{C}}$ for any $\lambda \in \mathbb{R}$ and $x \in[a, b]$.

Proof. (i) This is because $\Phi(\cdot, \lambda)$ is a fundamental solution of (2.9).

(ii) Under our assumptions, $F+(-i)^{n} \lambda W$ is $\mathscr{G}_{n}^{\mathbb{C}}$-valued for every $\lambda \in \mathbb{R}$. Thus, the claim follows from the fact that $\Phi(a, \lambda)=I \in \mathbf{G}_{n}^{\mathbb{C}}$.

Now, we start our discussions on various sets of BCs having certain eigenvalues. First, generalizing [15, Theorem 4.1], we have the following result.

TheOREM 4.3. Let $\lambda \in \mathbb{C}$. Then, among all the complex boundary conditions, $[\Phi(b$, $\lambda) \mid-I]$ is the unique one that has $\lambda$ as an eigenvalue of geometric multiplicity $n$.

Proof. The proof is very similar to that of [15, Theorem 4.1], and hence we omit it.

We will call the complex curve

$$
\lambda \longmapsto[\Phi(b, \lambda) \mid-I], \quad \lambda \in \mathbb{C},
$$

in $\mathcal{N}_{N}^{\mathbb{C}} \subset \mathscr{A}^{\mathbb{C}}$ the complex characteristic curve for the QDE (2.6) and denote it by $\mathscr{D}^{\mathbb{C}}$, where $N=\{n+1, n+2, \ldots, 2 n\}$, while the analytic real curve

$$
\lambda \longmapsto[\Phi(b, \lambda) \mid-I], \quad \lambda \in \mathbb{R}
$$

will be called the real characteristic curve for the QDE and given the notation $\mathscr{D}^{\mathbb{R}}$. Note that $\mathscr{D}^{\mathbb{R}} \subset \mathcal{N}_{N}^{\mathbb{R}} \subset \mathscr{A}^{\mathbb{R}}$ if $F+(-i)^{n} \lambda W$ is real-valued for every $\lambda \in \mathbb{R}$. Moreover, $\mathscr{D}^{\mathbb{R}} \subset \mathcal{O}_{N}^{\mathbb{C}} \subset$ $\mathscr{B}^{\mathbb{C}}$ when $F$ is $E$-symmetric and $w$ is real-valued.

Theorem 4.3 implies that any complex BC not on $\mathscr{D}^{\mathbb{C}}$ only has eigenvalues of geometric multiplicities less than or equal to $n-1$. Note that $\mathscr{A}^{\mathbb{C}}$ has complex dimension $n^{2}$, while $\mathscr{D}^{\mathbb{C}} \subset \mathscr{A}^{\mathbb{C}}$ is just an analytic subset of complex dimension 1 . So, it is very rare for a complex BC to have an eigenvalue of geometric multiplicity $n$. Moreover, $\mathscr{B}^{\mathbb{C}}$ has (real) dimension $n^{2} \geq 4$. Thus, it is also very rare for a complex selfadjoint BC to have an eigenvalue of geometric multiplicity $n$. Similarly, since $\mathscr{B}^{\mathbb{R}}$ has dimension $2 k^{2}+k \geq 3$ if $n=2 k$ or $n=2 k+1$, it is still very rare for a real selfadjoint BC to have an eigenvalue of geometric multiplicity $n$.

Next, we want to determine all the complex BCs that have a fixed $\lambda \in \mathbb{C}$ as an eigenvalue. Let $\mathscr{E}_{\lambda}^{\mathbb{C}}$ be the set of these BCs, that is,

$$
\mathscr{E}_{\lambda}^{\mathbb{C}}=\left\{[A \mid B] \in \mathscr{A}^{\mathbb{C}} ; \operatorname{det}(A+B \Phi(b, \lambda))=0\right\} .
$$

Then, $\mathscr{E}_{\lambda}^{\mathbb{R}}$ has its obvious meaning. For $\mathbb{K}=\mathbb{C}$ or $\mathbb{R}$ and $\lambda \in \mathbb{K}$, we will call $\mathscr{C}_{\lambda}^{\mathbb{K}}$ the $\lambda$ hypersurface in $\mathscr{A}^{\mathbb{K}}$. When $\lambda \in \mathbb{R}$ such that $\Phi(b, \lambda)$ is real,

$$
\begin{aligned}
\mathscr{E}_{\lambda}^{\mathbb{R}} & =\mathscr{E}^{\mathbb{R}} \cdot \Phi(b, \lambda)=\left\{[A \Phi(b, \lambda) \mid B] ;[A \mid B] \in \mathscr{E}^{\mathbb{R}}\right\} \\
& =\left\{\left[A \mid B \Phi(b, \lambda)^{-1}\right] ;[A \mid B] \in \mathscr{E}^{\mathbb{R}}\right\},
\end{aligned}
$$


where

$$
\mathscr{C}^{\mathbb{R}}=\left\{[A \mid B] \in \mathscr{A}^{\mathbb{R}} ; \operatorname{det}(A+B)=0\right\} .
$$

Similarly, for any $\lambda \in \mathbb{C}$,

$$
\begin{aligned}
\mathscr{E}_{\lambda}^{\mathbb{C}} & =\mathscr{E} \mathbb{C} . \Phi(b, \lambda)=\{[A \Phi(b, \lambda) \mid B] ;[A \mid B] \in \mathscr{E} \mathbb{C}\} \\
& =\left\{\left[A \mid B \Phi(b, \lambda)^{-1}\right] ;[A \mid B] \in \mathscr{E} \mathbb{C}\right\},
\end{aligned}
$$

where

$$
\mathscr{E}^{\mathbb{C}}=\left\{[A \mid B] \in \mathscr{A}^{\mathbb{C}} ; \operatorname{det}(A+B)=0\right\} .
$$

Therefore, we have proven the following generalization of [15, Theorem 4.3].

THEOREM 4.4. (i) The complex characteristic curve determines all the eigenvalues for each complex boundary condition in the explicit manner given in (4.22) and (4.23); when $F+(-i)^{n} \lambda W$ is real-valued for every $\lambda \in \mathbb{R}$, the real characteristic surface determines all the real eigenvalues for each real boundary condition in the explicit manner given in (4.20) and (4.21).

(ii) Each $\mathscr{E}_{\lambda}^{\mathbb{C}}$ is the image of $\mathscr{E}^{\mathbb{C}}$ under a diffeomorphism of $\mathscr{A}^{\mathbb{C}}$ given by a Lie group action, which sends $\mathscr{E}^{\mathbb{R}}$ to the corresponding $\mathscr{E}_{\lambda}^{\mathbb{R}}$ when both $\lambda$ and $\Phi(b, \lambda)$ are real.

REMARK 4.5. From the point of view of differential topology, the subsets $\mathscr{E}_{\lambda}^{\mathbb{C}}, \lambda \in \mathbb{C}$, of $\mathscr{A}^{\mathbb{C}}$ are the same as $\mathscr{E}^{\mathbb{C}}$. This means that the shapes of the sets $\mathscr{E}_{\lambda}^{\mathbb{C}}$ do not depend on the actual QDE in question. There are similar statements about the subsets $\mathscr{E}_{\lambda}^{\mathbb{R}}$, where $\lambda \in \mathbb{R}$ such that $\Phi(b, \lambda)$ is real, of $\mathscr{A}^{\mathbb{R}}$.

REMARK 4.6. The subsets $\mathscr{E}_{\lambda}^{\mathbb{C}}, \lambda \in \mathbb{C}$, of $\mathscr{A}^{\mathbb{C}}$ are solely determined by $\Phi(b, \lambda)$, and no more information about the QDE is needed. Moreover, the way in which $\Phi(b, \lambda)$ determines $\mathscr{E}_{\lambda}^{\mathbb{C}}$ is independent of the QDE in question. In other words, the eigenvalues for the complex BCs are determined by the QDE via an intermediate and geometric object-the complex characteristic curve $\mathscr{D}^{\mathbb{C}}$. There are similar statements about the subsets $\mathscr{E}_{\lambda}^{\mathbb{R}}, \lambda \in \mathbb{R}$, of $\mathscr{A}^{\mathbb{R}}$ and the real characteristic curve $\mathscr{D}^{\mathbb{R}}$ when $F+(-i)^{n} \lambda W$ is real-valued for every $\lambda \in \mathbb{R}$. These observations imply the following generalization of [15, Corollary 4.6].

COROLlary 4.7. (i) Let $\lambda_{*}$ and $\lambda_{\#}$ be two complex numbers. If there is a complex boundary condition having $\lambda_{*}$ and $\lambda_{\#}$ as eigenvalues of geometric multiplicity $n$, then any complex boundary condition having one of $\lambda_{*}$ and $\lambda_{\#}$ as an eigenvalue must have both of them as eigenvalues.

(ii) When $F+(-i)^{n} \lambda W$ is real-valued for every $\lambda \in \mathbb{R}$, the result in (i) is still true if only real boundary conditions and real eigenvalues are considered.

For an example illustrating this corollary, see [15, example after Corollary 4.6].

REMARK 4.8. Theorem 4.4 raises the following question: how can one determine the QDE (2.6), that is, its coefficient function matrix $F$ and its weight function $w$, using the geometric properties of the complex or real characteristic curve? 
REMARK 4.9. Note that each $\mathscr{E}_{\lambda}^{\mathbb{C}}$ is an algebraic variety in $\mathscr{A}^{\mathbb{C}}=\mathrm{G}_{2 n, n}(\mathbb{C})$. Thus, if $2 \leq i<2 n^{2}$ and $G_{1}, \ldots, G_{i}$ are in $\operatorname{GL}(n, \mathbb{C})$, then the intersection of $\mathscr{C}_{\bullet} \mathbb{C} G_{1}, \ldots, \mathscr{E} \mathbb{C} G_{i}$ is generically of (real) dimension $2 n^{2}-i-1$; if $G_{1}, G_{2}, \ldots$, and $G_{2 n^{2}}$ are in $\operatorname{GL}(n, \mathbb{C})$, then the intersection of $\mathscr{E} \mathbb{C}, G_{1}, \mathscr{E} \mathbb{C}, G_{2}, \ldots$, and $\mathscr{E}^{\mathbb{C}} \cdot G_{2 n^{2}}$ is generically empty. Hence, given a QDE, it is very "rare" for a fixed set of $2 n^{2}$ complex numbers to be eigenvalues of a complex BC at the same time. Similarly, given a QDE with $F+(-i)^{n} \lambda W$ being realvalued for every $\lambda \in \mathbb{R}$, it is also very "rare" for a fixed set of $n^{2}+1$ real numbers to be eigenvalues of a real $\mathrm{BC}$ at the same time.

Now, we give a topological description of the sets $\mathscr{E}_{\lambda}^{\mathbb{C}} \subset \mathscr{A}^{\mathbb{C}}, \lambda \in \mathbb{C}$, and $\mathscr{E}_{\lambda}^{\mathbb{R}} \subset \mathscr{A}^{\mathbb{R}}$, $\lambda \in \mathbb{R}$. For this purpose, we only need to look at $\mathscr{E} \mathbb{C}$ and $\mathscr{E} \mathbb{R}$, by (4.20) and (4.22).

We first consider $\mathscr{E}^{\mathbb{R}}$. Note that

$$
\mathscr{E}^{\mathbb{R}} \cap \mathcal{N}_{n+1, n+2, \ldots, 2 n}^{\mathbb{R}}=\left\{[A+I \mid-I] ; A \in \mathrm{M}_{n, n}(\mathbb{R}), \operatorname{det} A=0\right\}
$$

can be regarded as a cone in $\mathrm{M}_{n, n}(\mathbb{R})$ with vertex $A=0$ and generating set

$$
\mathscr{F}^{\mathbb{R}}=\left\{A \in \mathrm{M}_{n, n}(\mathbb{R}) ; 1 \leq \operatorname{rank} A \leq n-1,\|A\|_{\mathbb{R}^{n^{2}}}=1\right\},
$$

that is,

$$
\mathscr{C}^{\mathbb{R}} \cap \mathcal{N}_{n+1, n+2, \ldots, 2 n}^{\mathbb{R}}=\left\{[\xi A+I \mid-I] ; A \in \mathscr{F}^{\mathbb{R}}, \xi \geq 0\right\} .
$$

Let $1 \leq m \leq n-1$. The matrices in $\mathrm{M}_{n, n}(\mathbb{R})$ with rank $m$ can be divided into disjoint subcollections parameterized by the subspace of $\mathbb{R}^{n}$ spanned by the rows of such a matrix. Fix an orthonormal basis $\left\{\mathbf{v}_{1}, \ldots, \mathbf{v}_{m}\right\}$ of an $m$-dimensional subspace $V$ of $\mathbb{R}^{n}$ consisting of row vectors. For any $m$ linearly independent column vectors

$$
\mathbf{c}_{1}=\left(\begin{array}{c}
c_{11} \\
c_{21} \\
\vdots \\
c_{n 1}
\end{array}\right), \ldots, \mathbf{c}_{m}=\left(\begin{array}{c}
c_{1 m} \\
c_{2 m} \\
\vdots \\
c_{n m}
\end{array}\right),
$$

we have an element

$$
\left(\begin{array}{c}
c_{11} \mathbf{v}_{1}+\cdots+c_{1 m} \mathbf{v}_{m} \\
c_{21} \mathbf{v}_{1}+\cdots+c_{2 m} \mathbf{v}_{m} \\
\vdots \\
c_{n 1} \mathbf{v}_{1}+\cdots+c_{n m} \mathbf{v}_{m}
\end{array}\right)
$$

of the subcollection corresponding to $V$, and every element of the subcollection can be uniquely written in this form. To get a linearly independent vector, just pick a nonzero vector; to get two linearly independent vectors, first take a nonzero vector $\mathbf{c}_{1}$ and then pick a nonzero vector not in the subspace spanned by $\mathbf{c}_{1}$, and so on so forth. The square of the norm of the element given by (4.28) is $\left\|\mathbf{c}_{1}\right\|_{\mathbb{R}^{n}}^{2}+\cdots+\left\|\mathbf{c}_{m}\right\|_{\mathbb{R}^{n}}^{2}$. For $1 \leq l \leq n-2$, topologically,

$$
\mathbb{S}^{n-1} \backslash \mathbb{R}^{l}=\mathbb{S}^{n-1} \backslash \mathbb{S}^{l-1} \simeq \mathbb{R}^{n-1} \backslash \mathbb{R}^{l-1} \simeq \mathbb{S}^{n-1-l} \times \mathbb{R}^{+} \times \mathbb{R}^{l-1} \simeq \mathbb{S}^{n-l-1} \times \mathbb{R}^{l} .
$$


Thus, topologically,

$$
\begin{aligned}
\mathscr{F}^{\mathbb{R}} \simeq & \mathrm{G}_{1}\left(\mathbb{R}^{n}\right) \times \mathbb{S}^{n-1} \\
& \cup \mathrm{G}_{2}\left(\mathbb{R}^{n}\right) \times \mathbb{S}^{n-1} \times \mathbb{R}^{+} \times\left(\mathbb{S}^{n-1} \backslash \mathbb{R}\right) \\
& \cup \mathrm{G}_{3}\left(\mathbb{R}^{n}\right) \times \mathbb{S}^{n-1} \times \mathbb{R}^{+} \times\left(\mathbb{S}^{n-1} \backslash \mathbb{R}\right) \times \mathbb{R}^{+} \times\left(\mathbb{S}^{n-1} \backslash \mathbb{R}^{2}\right) \\
& \cup \cdots \cup \mathrm{G}_{n-1}\left(\mathbb{R}^{n}\right) \times \mathbb{S}^{n-1} \times \mathbb{R}^{+} \times\left(\mathbb{S}^{n-1} \backslash \mathbb{R}\right) \\
& \times \mathbb{R}^{+} \times \cdots \times\left(\mathbb{S}^{n-1} \backslash \mathbb{R}^{n-3}\right) \times \mathbb{R}^{+} \times\left(\mathbb{S}^{n-1} \backslash \mathbb{R}^{n-2}\right) \\
\simeq & \mathrm{G}_{1}\left(\mathbb{R}^{n}\right) \times \mathbb{S}^{n-1} \\
& \cup \mathrm{G}_{2}\left(\mathbb{R}^{n}\right) \times \mathbb{S}^{n-1} \times \mathbb{S}^{n-2} \times \mathbb{R}^{2} \\
& \cup \mathrm{G}_{3}\left(\mathbb{R}^{n}\right) \times \mathbb{S}^{n-1} \times \mathbb{S}^{n-2} \times \mathbb{S}^{n-3} \times \mathbb{R}^{5} \\
& \cup \cdots \cup \mathrm{G}_{n-1}\left(\mathbb{R}^{n}\right) \times \mathbb{S}^{n-1} \times \cdots \times \mathbb{S}^{1} \times \mathbb{R}^{\left(n^{2}-n-2\right) / 2} .
\end{aligned}
$$

Note also that $\mathscr{E}^{\mathbb{R}} \backslash \mathcal{N}_{n+1, n+2, \ldots, 2 n}^{\mathbb{R}}$ has a dimension less than that of $\mathscr{C}^{\mathbb{R}}$. For example,

$$
\mathscr{C}^{\mathbb{R}} \cap\left(\mathcal{N}_{1,2, \ldots, n}^{\mathbb{R}} \backslash \mathcal{N}_{n+1, n+2, \ldots, 2 n}^{\mathbb{R}}\right)=\left\{[I \mid B] ; B \in \mathrm{M}_{n, n}(\mathbb{R}), \operatorname{det} B=0, \operatorname{det}(I+B)=0\right\} ;
$$

since $\operatorname{det} B=0$ and $\operatorname{det}(I+B) \neq 0$ when $B=0$, while $\operatorname{det} B \neq 0$ and $\operatorname{det}(I+B)=0$ when $B=-I$, we see that none of the polynomials $\operatorname{det} B$ and $\operatorname{det}(I+B)$ is a factor of the other, and hence the part of $\mathscr{E}^{\mathbb{R}} \backslash \mathcal{N}_{n+1, n+2, \ldots, 2 n}^{\mathbb{R}}$ in $\mathcal{N}_{1,2, \ldots, n}^{\mathbb{R}}$ has a dimension less than that of $\mathscr{E} \mathbb{R}$. Thus, $\mathscr{E}^{\mathbb{R}} \backslash \mathcal{N}_{n+1, n+2, \ldots, 2 n}^{\mathbb{R}}$ is in the limit set of $\mathscr{E}^{\mathbb{R}} \cap \mathcal{N}_{n+1, n+2, \ldots, 2 n}^{\mathbb{R}}$, that is,

$$
\mathscr{E}^{\mathbb{R}} \backslash \mathcal{N}_{n+1, n+2, \ldots, 2 n}^{\mathbb{R}}=\left\{\lim _{\xi \rightarrow+\infty}[\xi A+I \mid-I] ; A \in \mathscr{F}_{\mathbb{R}}^{\mathbb{R}}\right\} .
$$

Let $A$ be the matrix given by (4.28) and assume that its $i_{1}$ th row $\mathbf{a}_{i_{1}}, \ldots, i_{m}$ th row $\mathbf{a}_{i_{m}}$ are linearly independent. Then, there is a matrix $C^{\perp} \in \mathrm{M}_{n-m, n}^{*}$ consisting of row vectors annihilating $\mathbf{c}_{1}, \ldots, \mathbf{c}_{m}$ (corresponding to row operations bringing the remaining $n-m$ rows of $A$ all to 0 ) such that for any $\xi \in \mathbb{R}$,

$$
[\xi A+I \mid-I]=\left[\begin{array}{c|c}
\xi \mathbf{a}_{i_{1}}+\mathbf{i}_{i_{1}} & -\mathbf{i}_{i_{1}} \\
\vdots & \vdots \\
\xi \mathbf{a}_{i_{m}}+\mathbf{i}_{i_{m}} & -\mathbf{i}_{i_{m}} \\
C^{\perp} & -C^{\perp}
\end{array}\right]
$$

where $\mathbf{i}_{i}$ is the $i$ th row of $I$ for $1 \leq i \leq n$. Thus,

$$
\lim _{\xi \rightarrow+\infty}[\xi A+I \mid-I]=\left[\begin{array}{c|c}
\mathbf{a}_{i_{1}} & 0 \\
\vdots & \vdots \\
\mathbf{a}_{i_{m}} & 0 \\
C^{\perp} & -C^{\perp}
\end{array}\right]=\left[\begin{array}{c|c}
\mathbf{v}_{1} & 0 \\
\vdots & \vdots \\
\mathbf{v}_{m} & 0 \\
C^{\perp} & -C^{\perp}
\end{array}\right],
$$

that is, the limit is uniquely determined by the subspaces $V$ and $\operatorname{span}_{\mathbb{R}}\left\{\mathbf{c}_{1}, \ldots, \mathbf{c}_{m}\right\}$. Hence, topologically,

$$
\mathscr{E}^{\mathbb{R}} \backslash \mathcal{N}_{n+1, n+2, \ldots, 2 n}^{\mathbb{R}} \simeq \mathrm{G}_{1}\left(\mathbb{R}^{n}\right)^{2} \cup \mathrm{G}_{2}\left(\mathbb{R}^{n}\right)^{2} \cup \cdots \cup \mathrm{G}_{n-1}\left(\mathbb{R}^{n}\right)^{2} .
$$


Next, we consider $\mathscr{E} \mathbb{C}$. As above,

$$
\mathscr{E}^{\mathbb{C}} \cap \mathcal{N}_{n+1, n+2, \ldots, 2 n}^{\mathbb{C}}=\left\{[A+I \mid-I] ; A \in \mathrm{M}_{n, n}(\mathbb{C}), \operatorname{det} A=0\right\}
$$

can be regarded as a cone in $\mathrm{M}_{n, n}(\mathbb{C})$ with vertex $A=0$ and generating set

$$
\mathscr{F}^{\mathbb{C}}=\left\{A \in \mathrm{M}_{n, n}(\mathbb{C}) ; 1 \leq \operatorname{rank} A \leq n-1,\|A\|_{\mathbb{C}^{n^{2}}}=1\right\},
$$

and topologically,

$$
\begin{aligned}
\mathscr{F}^{\mathbb{C}} \simeq & \mathrm{G}_{1}\left(\mathbb{C}^{n}\right) \times \mathbb{S}^{2 n-1} \\
& \cup \mathrm{G}_{2}\left(\mathbb{C}^{n}\right) \times \mathbb{S}^{2 n-1} \times \mathbb{R}^{+} \times\left(\mathbb{S}^{2 n-1} \backslash \mathbb{C}\right) \\
& \cup \mathrm{G}_{3}\left(\mathbb{C}^{n}\right) \times \mathbb{S}^{2 n-1} \times \mathbb{R}^{+} \times\left(\mathbb{S}^{2 n-1} \backslash \mathbb{C}\right) \times \mathbb{R}^{+} \times\left(\mathbb{S}^{2 n-1} \backslash \mathbb{C}^{2}\right) \\
& \cup \cdots \cup \mathrm{G}_{n-1}\left(\mathbb{C}^{n}\right) \times \mathbb{S}^{2 n-1} \times \mathbb{R}^{+} \times\left(\mathbb{S}^{2 n-1} \backslash \mathbb{C}\right) \\
& \times \mathbb{R}^{+} \times \cdots \times\left(\mathbb{S}^{2 n-1} \backslash \mathbb{C}^{n-3}\right) \times \mathbb{R}^{+} \times\left(\mathbb{S}^{2 n-1} \backslash \mathbb{C}^{n-2}\right) \\
\simeq & \mathrm{G}_{1}\left(\mathbb{C}^{n}\right) \times \mathbb{S}^{2 n-1} \\
& \cup \mathrm{G}_{2}\left(\mathbb{C}^{n}\right) \times \mathbb{S}^{2 n-1} \times \mathbb{S}^{2 n-3} \times \mathbb{R}^{3} \\
& \cup \mathrm{G}_{3}\left(\mathbb{C}^{n}\right) \times \mathbb{S}^{2 n-1} \times \mathbb{S}^{2 n-3} \times \mathbb{S}^{2 n-5} \times \mathbb{R}^{8} \\
& \cup \cdots \cup \mathrm{G}_{n-1}\left(\mathbb{C}^{n}\right) \times \mathbb{S}^{2 n-1} \times \cdots \times \mathbb{S}^{3} \times \mathbb{R}^{n^{2}-2 n} .
\end{aligned}
$$

For a topological manifold $M$, a topological $M$-bottle is a quotient space $N$ that one obtains from $M \times[0,1]$ via modeling $M \times\{0\}$ by an equivalence relation on $M$ to form a subset of $N$, to be called the top of $N$, and modeling $M \times\{1\}$ by another equivalence relation on $M$ to form a topological submanifold of $N$, to be called the bottom of $N$. In this case, $M \times(0,1)$ is called the side of $N$. With the concept of topological bottle in hand, what we have proven above can be summarized in the following theorem.

THEOREM 4.10. (i) The hypersurface $\mathscr{E}^{\mathbb{R}}$ in $\mathbb{A}^{\mathbb{R}}$ is a topological $\mathscr{F}^{\mathbb{R}}$-bottle with a point top and a bottom given by (4.35), while the map gluing its side to its bottom is specified by (4.34). Moreover, a topological description of $\mathscr{F}^{\mathbb{R}}$ is given by (4.30).

(ii) The complex hypersurface $\mathscr{E}^{\mathbb{C}}$ in $\mathscr{A}^{\mathbb{C}}$ is a topological $\mathscr{F}^{\mathbb{C}}$-bottle with a point top and a bottom

$$
\mathscr{E}^{\mathbb{C}} \backslash \mathcal{N}_{n+1, n+2, \ldots, 2 n}^{\mathbb{C}} \simeq \mathrm{G}_{1}\left(\mathbb{C}^{n}\right)^{2} \cup \mathrm{G}_{2}\left(\mathbb{C}^{n}\right)^{2} \cup \cdots \cup \mathrm{G}_{n-1}\left(\mathbb{C}^{n}\right)^{2},
$$

while the map gluing its side to its bottom is also specified by (4.34). Moreover, a topological description of $\mathscr{F}^{\mathbb{C}}$ is given by (4.38).

Finally, for each real number $\lambda$, we can consider the subset of BCs in $\mathscr{B}^{\mathbb{R}}$ (or $\mathscr{B}^{\mathbb{C}}$ ) that have $\lambda$ as an eigenvalue, to be called the $\lambda$-subset in $\mathscr{B}^{\mathbb{R}}$ (or $\mathscr{B}^{\mathbb{C}}$ ). When $n$ is odd, we define the $\lambda$-subsets in $\mathscr{B}_{-}^{\mathbb{R}}$ and $\mathscr{B}_{+}^{\mathbb{R}}$ similarly, and each $\lambda$-subset in $\mathscr{B}^{\mathbb{R}}$ is the union of the corresponding $\lambda$-subsets in $\mathscr{B}_{-}^{\mathbb{R}}$ and $\mathscr{B}_{+}^{\mathbb{R}}$. When $F$ is $E$-symmetric and $w$ is real-valued, by Proposition 4.2(ii), we can characterize the $\lambda$-subsets in $\mathscr{B}^{\mathbb{C}}$ using $\mathscr{E}^{\mathbb{C}} \cap \mathscr{B}^{\mathbb{C}}$ just as we characterized $\mathscr{E}_{\lambda}^{\mathbb{C}}$ using $\mathscr{E} \mathbb{C}$. There is a similar statement for the $\lambda$-subsets in $\mathscr{B}^{\mathbb{R}}$ when $n$ is even. It is quite involved to characterize the $\lambda$-subsets in $\mathscr{B}^{\mathbb{R}}$ when $n$ is odd, and 
it is even more involved to describe the $\lambda$-subsets in $\mathscr{B}^{\mathbb{C}}$ or $\mathscr{B}^{\mathbb{R}}$ topologically. We plan to do these in a forthcoming publication. Here we only give an example showing that when $n$ is odd, the $\lambda$-subsets in $\mathscr{B}_{+}^{\mathbb{R}}$ are complicated in general, even their dimensions can differ for different values of $\lambda$.

EXAMPLE 4.11. Let $n=3$. Consider the QDE

$$
Q y=\lambda y \quad \text { on }(0,1)
$$

with $E$-symmetric coefficient matrix

$$
F=\left(\begin{array}{lll}
0 & 1 & 0 \\
0 & 0 & 1 \\
0 & 0 & 0
\end{array}\right)
$$

Since $F$ is real, so is $\Phi(1,0)$. Thus, by Proposition $4.1,\left(\mathscr{R}_{+}^{\mathbb{R}}\right) . \Phi(1,0)=\mathscr{B}_{+}^{\mathbb{R}}$. Hence, by Theorem 2.3, 0 is an eigenvalue for each selfadjoint $\mathrm{BC}$ in $\mathscr{B}_{+}^{\mathbb{R}}$, that is, the 0 -subset in $\mathscr{B}_{+}^{\mathbb{R}}$ is $\mathscr{B}_{+}^{\mathbb{R}}$ and hence has dimension 3. Direct calculations show that the characteristic function for the general $B C$

$$
\left[\begin{array}{cccccc}
1 & 0 & -\frac{1}{2} d^{2} & 0 & d & -c-d e \\
0 & 1 & -d & 0 & 1 & -e \\
0 & 0 & c & -1 & e & -\frac{1}{2} e^{2}
\end{array}\right]
$$

in $\mathscr{B}_{+}^{\mathbb{R}}$ is

$$
\Delta(\lambda)=0+p(c, d, e) \lambda+\text { higher terms in } \lambda,
$$

where $p$ is a polynomial of degree 4 . So, when $\lambda \neq 0$ is real and sufficiently close to 0 , the $\lambda$-subset in $\mathscr{B}_{+}^{\mathbb{R}}$ has a dimension less than or equal to 2 .

ACKNOWLEDGMENTS. The first author is supported by the National Natural Science Foundation of China through the Grant 10226037 and the second author is supported by the National Science Foundation through the Grant DMS-9973108. Part of this work was done during a visit of the first author to the Mathematics Department at Concordia University, and he would like to thank the department for its hospitality.

\section{REFERENCES}

[1] X. Cao, Q. Kong, H. Wu, and A. Zettl, Sturm-Liouville problems whose leading coefficient function changes sign, Canad. J. Math. 55 (2003), no. 4, 724-749.

[2] N. Dunford and J. T. Schwartz, Linear Operators. Part II: Spectral Theory. Self Adjoint Operators in Hilbert Space, Interscience Publishers, New York, 1963.

[3] M. S. P. Eastham, Q. Kong, H. Wu, and A. Zettl, Inequalities among eigenvalues of SturmLiouville problems, J. Inequal. Appl. 3 (1999), no. 1, 25-43.

[4] W. N. Everitt and L. Markus, The Glazman-Krein-Naimark theorem for ordinary differential operators, New Results in Operator Theory and Its Applications, Oper. Theory Adv. Appl., vol. 98, Birkhäuser, Basel, 1997, pp. 118-130. 
[5] _ Boundary Value Problems and Symplectic Algebra for Ordinary Differential and Quasi-Differential Operators, Mathematical Surveys and Monographs, vol. 61, American Mathematical Society, Rhode Island, 1999.

[6] _ Complex symplectic geometry with applications to ordinary differential operators, Trans. Amer. Math. Soc. 351 (1999), no. 12, 4905-4945.

[7] W. N. Everitt and A. Zettl, Generalized symmetric ordinary differential expressions. I. The general theory, Nieuw Arch. Wisk. (3) 27 (1979), no. 3, 363-397.

[8] _ Differential operators generated by a countable number of quasi-differential expressions on the real line, Proc. London Math. Soc. (3) 64 (1992), no. 3, 524-544.

[9] K. Haertzen, Q. Kong, H. Wu, and A. Zettl, Geometric aspects of Sturm-Liouville problems. II. Space of boundary conditions for left-definiteness, Trans. Amer. Math. Soc. 356 (2004), no. 1, 135-157.

[10] S. Kobayashi and K. Nomizu, Foundations of Differential Geometry. Vol. I, Interscience Publishers, New York, 1963.

[11] _ Foundations of Differential Geometry. Vol. II, Interscience Tracts in Pure and Applied Mathematics, no. 15, Interscience Publishers, New York, 1969.

[12] Q. Kong, Q. Lin, H. Wu, and A. Zettl, A new proof of the inequalities among Sturm-Liouville eigenvalues, Panamer. Math. J. 10 (2000), no. 2, 1-11.

[13] Q. Kong, H. Wu, and A. Zettl, Dependence of eigenvalues on the problem, Math. Nachr. 188 (1997), 173-201.

[14] _ Dependence of the nth Sturm-Liouville eigenvalue on the problem, J. Differential Equations 156 (1999), no. 2, 328-354.

[15] _ Geometric aspects of Sturm-Liouville problems. I. Structures on spaces of boundary conditions, Proc. Roy. Soc. Edinburgh Sect. A 130 (2000), no. 3, 561-589.

[16] Q. Kong and A. Zettl, Eigenvalues of regular Sturm-Liouville problems, J. Differential Equations 131 (1996), no. 1, 1-19.

[17] M. Möller, On the unboundedness below of the Sturm-Liouville operator, Proc. Roy. Soc. Edinburgh Sect. A 129 (1999), no. 5, 1011-1015.

[18] M. A. Naumark, Linear Differential Operators. Part II: Linear Differential Operators in Hilbert Space, Frederick Ungar Publishing, New York, 1968, Translated from Russian by E. R. Dawson.

[19] J. Weidmann, Spectral Theory of Ordinary Differential Operators, Lecture Notes in Mathematics, vol. 1258, Springer-Verlag, Berlin, 1987.

[20] A. Zettl, Formally self-adjoint quasi-differential operators, Rocky Mountain J. Math. 5 (1975), 453-474.

Xifang Cao: Department of Mathematics, Yangzhou University, Yangzhou, Jiangsu 225002, China

E-mail address: xifangcao@yahoo.com

Hongyou Wu: Department of Mathematical Sciences, Northern Illinois University, DeKalb, IL 60115, USA

E-mail address: wu@math.niu.edu 


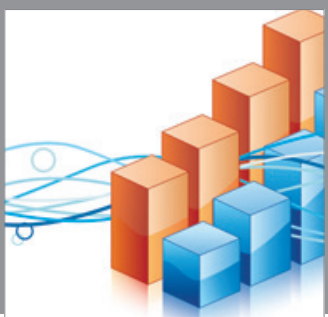

Advances in

Operations Research

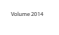

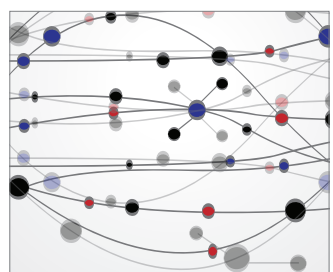

\section{The Scientific} World Journal
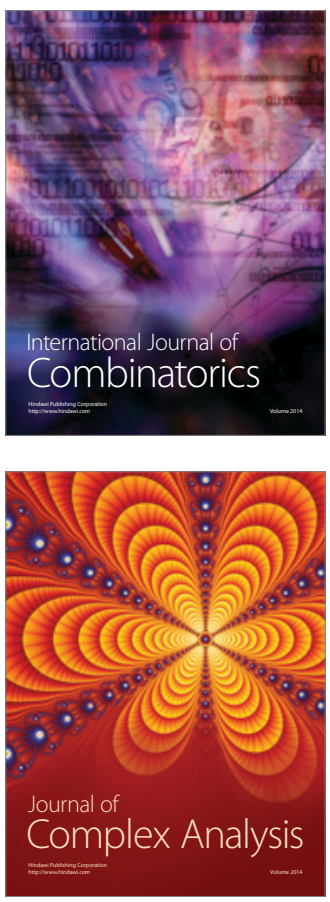

International Journal of

Mathematics and

Mathematical

Sciences
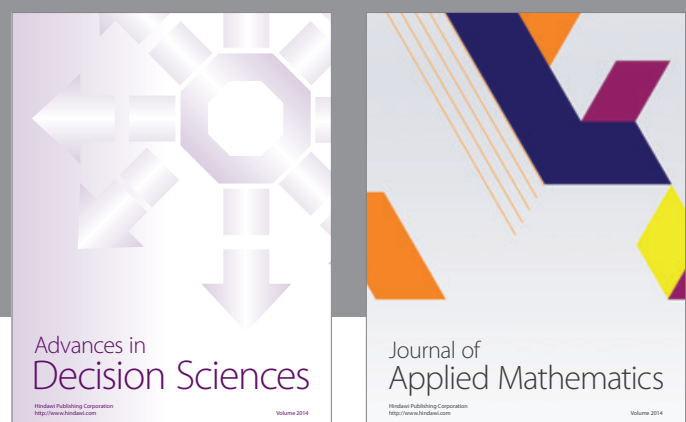

Journal of

Applied Mathematics
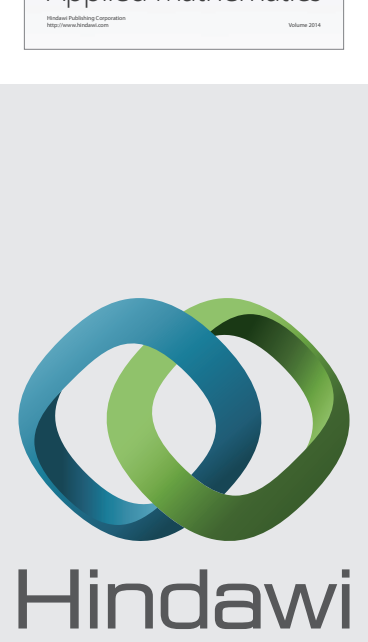

Submit your manuscripts at http://www.hindawi.com
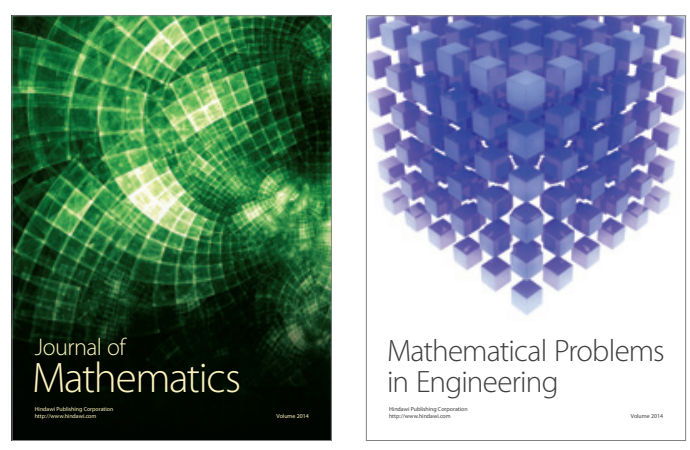

Mathematical Problems in Engineering
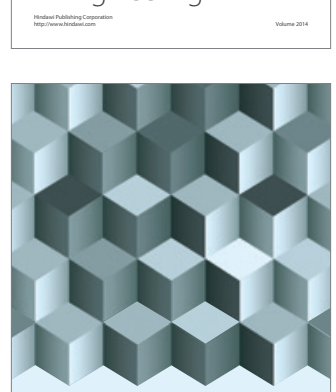

Journal of

Function Spaces
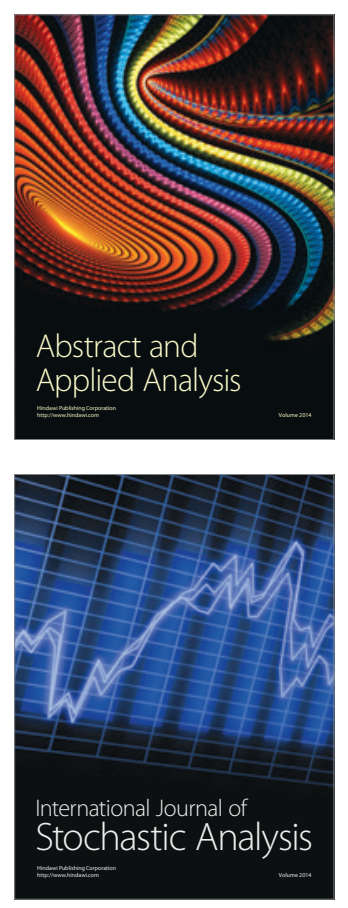

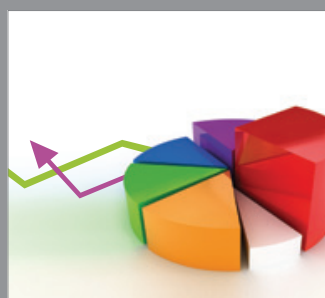

ournal of

Probability and Statistics

Promensencen
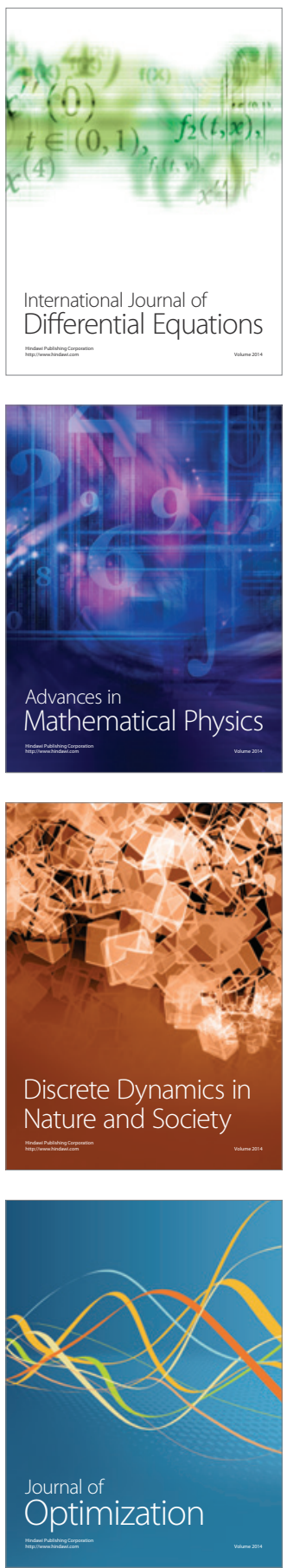